\title{
ESTIMATION OF DISPLACEMENT \\ LOCATION FOR ENHANCED \\ STRAIN IMAGING
}

J. E. Lindop, G. M. Treece, A. H. Gee and R. W. Prager

\section{CUED/F-INFENG/TR 550}

March 2006

\footnotetext{
University of Cambridge

Department of Engineering

Trumpington Street

Cambridge CB2 1PZ

United Kingdom
}

Email: jel35/gmt11/ahg/rwp @eng.cam.ac.uk 


\title{
ESTIMATION OF DISPLACEMENT LOCATION FOR ENHANCED STRAIN IMAGING
}

\author{
Joel E. Lindop, Graham M. Treece, Andrew H. Gee and Richard W. Prager \\ University of Cambridge \\ Department of Engineering \\ Trumpington Street \\ Cambridge CB2 1PZ
}

\begin{abstract}
Ultrasonic strain imaging is usually based on displacement estimates computed using finitelength sections of the RF ultrasound signal. Amplitude variations in the ultrasound are known to cause a perturbation in the location at which the displacement estimate is valid. If this goes uncorrected, it is an important source of estimation noise, which is amplified when the displacement field is converted into a strain image. We present a study of this effect based on theoretical analysis and practical experiments. A correction method based on the analysis is tested on phase and correlation coefficient strain imaging, and compared to the log compression technique from an earlier study. The performance is also compared against adaptive strain estimation. Results indicate that the new correction yields a substantial reduction in estimation noise.
\end{abstract}

\section{Introduction}

Ultrasonic elasticity imaging spans a broad range of techniques that process ultrasound signals to extract information relating to tissue's mechanical properties. A majority of these techniques require high quality displacement tracking at the first stage of signal processing. Examples include quasistatic compression imaging [26, 29], axial shear wave imaging [32] and acoustic radiation force imaging in both quasistatic/impulsive [24] and dynamic [2] forms. The principal alternative, sonoelasticity imaging $[18,27]$, employs Doppler velocity estimation in mechanically vibrated tissues. This is a practical technique, although the images it yields are relatively difficult to interpret. Displacement-based imaging systems have been investigated for a wide range of diagnostic purposes, spanning screening for soft tissue tumours [7, 9, 28], monitoring of atherosclerosis [6], assessment of skin pathologies [8, 40] and examination of cardiac disease [15] among other applications. The simplest form of meaningful visualisation is the strain image. This is extended by some of the more complicated systems, where strain image sequences are analysed to infer material property estimates such as elastic $[12,32]$ and viscoelastic $[2,10]$ moduli.

The cornerstone of elasticity imaging - displacement tracking — is easily understood. Consider a pair of ultrasound frames recorded consecutively during a scan: we refer to them as the pre- and post-deformation frames. A window is placed around a point of interest in the pre-deformation frame, and the closest match in the post-deformation frame is located. In practice, this is an optimisation problem, where the peak must be found in some suitable measure of signal similarity, such as the correlation coefficient [20,26], sum of absolute (SAD) or squared (SSD) differences $[17,21,39]$ or mutual information [22]. Numerous phase-based approaches have also been developed $[5,25,29]$, which exploit a property of the cross-correlation function peak, and are advantageous because of relatively low computational cost. Whichever technique has been used to match the windows, it is usually assumed thereafter that the mechanical displacement of tissue at the centre of the window is equal to the optimal window displacement $[13,20,26,29,31]$. Window-matching is applied at positions throughout a grid over the acquired frame of ultrasound data, constructing a fine map of the displacement field. 


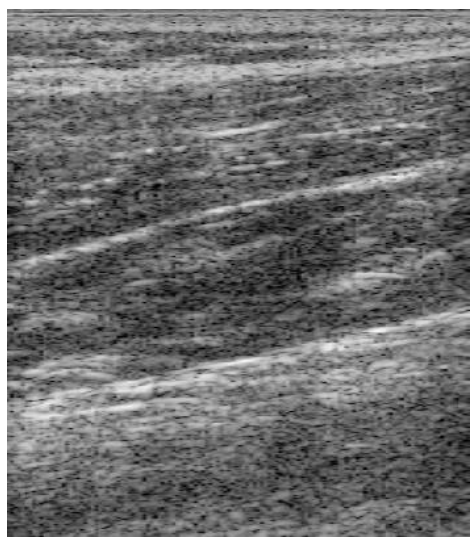

(a)

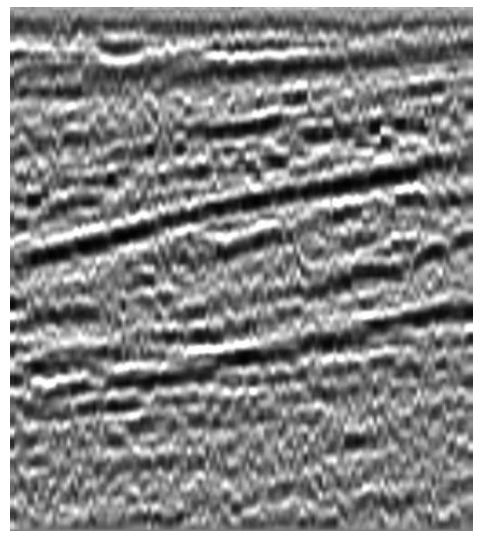

(b)

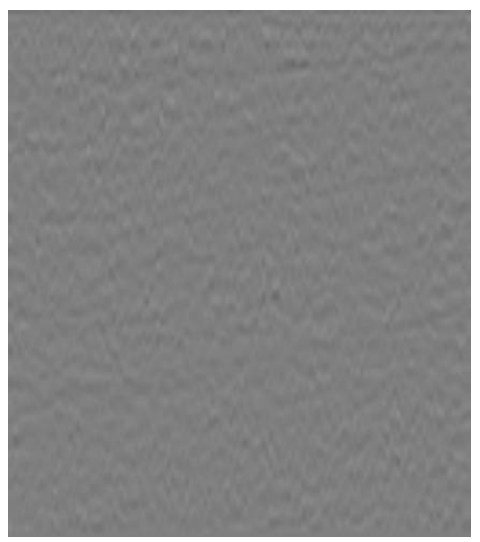

(c)

Figure 1: (a) B-mode image of RF data from a scan of human arm. The signal is temporally compressed to simulate a uniform compressive strain of $1 \%$. On a linear scale from black $(0 \%$ strain) to white $(2 \%)$, this should produce a uniform strain image with extremely low estimation noise, since the signal SNR is higher than could possibly be achieved in a real compression scan. However, (b) the standard correlation coefficient maximiser produces a strain image that is severely degraded (and misleading) owing to the AM effect, while (c) shows the (near perfect) result from applying the best of the correction techniques introduced in this paper. Strain estimation for both images used windows of length $13.5 \lambda$.

A strain image can be produced by displaying spatial derivatives from the estimated displacement field. In this paper we consider in detail the problem of axial strain image formation, although some of the principles we derive are more generally applicable. Strain estimation may be regarded as a stochastic process, in which case the terms "mean squared error", "estimation noise" and "estimation variance" may be used interchangeably when referring to the typical discrepancies between actual deformations and the estimates that are recorded and displayed. Errors in strain images arise mostly from two sources. The first is displacement estimation error, which is well understood. Following Carter [3] and Walker and Trahey [41, 42] it has become popular to apply Cramer-Rao lower bound analyses (and variations thereon) to signals with known properties, thereby identifying a lower bound on the displacement estimation variance that could be achieved by a maximum likelihood estimator [13, 14, 16, 36, 37, 38, 39].

Compared to displacement estimation error, the second source of noise has received little attention in the literature. The problem is estimation location variance: it is not generally true that the displacement estimate most closely tracks the actual displacement at the window centre. It was noted in an earlier study by Céspedes and Ophir [4] that if there is intra-window compression and the signal envelope is not constant, then the actual estimation location is skewed towards higher amplitude portions of the windowed signal. This causes artefacts at the boundaries between regions of differing echogenicity, as demonstrated by Figure 1. It is observed that strain estimates are corrupted by unwanted modulation from the amplitude, which we call the amplitude modulation (AM) effect. In fact, the AM effect also degrades strain estimates within regions that are isoechoic, since the signal returned from a fine scatterer distribution does not have a constant envelope. Nevertheless, the AM effect is most dangerous in anisoechoic regions, where AM noise correlates strongly with the features in B-mode images, and can easily lead to severe misinterpretations of strain images.

It will be shown in the following section that the AM effect is often the primary source of error in ultrasonic strain images where it is not corrected. Two correction techniques were proposed by Céspedes and Ophir [4]. Firstly, log compression of the signal envelope reduces amplitude fluctuations, thereby shifting estimation locations towards the window centres. This is an effective 
means of mitigating the AM effect, and has consequently been reapplied in more recent strain imaging systems [19, 29]. The second suggestion was adaptive stretching [4], which compensates for intra-window compression by stretching the signal to enable a close match to the true displacement at all points. This has been shown in numerous studies to be a good way of reducing strain estimation noise, although such techniques are computationally expensive [1,21, 22, 33]. The estimation location variance can also be reduced by using shorter estimation windows [20], but this is inevitably accompanied by reduced accuracy in the displacement estimates, since displacement estimation variance increases as the reciprocal of the window length.

The AM effect is present in all displacement tracking methods that use amplitude information, including methods based on the (normalised) correlation coefficient. To eliminate the AM effect, the amplitude must be entirely suppressed, as in one-bit compression, but this may bring unwanted side effects. The following section examines the AM effect from a theoretical standpoint, leading to a surprisingly simple AM correction method $\left(\mathrm{AMC}^{1}\right)$. Experiments have been performed using simulated RF ultrasound data to compare the performance of phase and correlation coefficient methods, and to evaluate the efficacy of correction by AMC, log compression and one-bit (limiting) compression in both cases. All of the corrections are computationally efficient and suitable for use in real-time imaging systems. Further experiments are performed using a direct strain estimator with adaptive stretching, which is slower but provides an AM suppression benchmark by which the other techniques may be judged.

\section{Amplitude modulation theory}

This section analyses the estimation of strain from a set of window displacement estimates. For the sake of clarity, we examine the simplest method for converting 1D displacement estimates to 1D strains, by taking the difference between displacements at consecutive windows, and dividing this by the spacing between the assumed estimation locations.

$$
\hat{s}=\frac{\hat{d}_{2}-\hat{d}_{1}}{\hat{\tau}_{2}-\hat{\tau}_{1}}
$$

$\hat{s}$ is the strain estimate, $\hat{d}_{1}$ and $\hat{d}_{2}$ are the displacement estimates for windows 1 and 2 respectively, and $\hat{\tau}_{1}$ and $\hat{\tau}_{2}$ are assumed to be the estimation locations. It is commonly assumed that Equation 1 contains only two random variables: $\hat{d}_{1}$ and $\hat{d}_{2}$. In this paper we examine the neglected variables, $\hat{\tau}_{2}$ and $\hat{\tau}_{1}$. New variables $\hat{D}$ and $\hat{F}$ are defined to simplify the strain calculation.

$$
\begin{aligned}
\hat{D} & =\hat{d}_{2}-\hat{d}_{1} \\
\hat{F} & =\frac{1}{\hat{\tau}_{2}-\hat{\tau}_{1}} \\
\hat{s} & =\hat{D} \hat{F}
\end{aligned}
$$

The sources of estimation noise are illustrated in Figure 2. We will assume that errors in $\hat{D}$ and in $\hat{F}$ are uncorrelated. This allows the strain estimation variance, $\sigma_{\hat{s}}^{2}$, to be expressed in a simple form.

$$
\sigma_{\hat{s}}^{2}=\sigma_{\hat{D}}^{2} \sigma_{\hat{F}}^{2}+\mu_{\hat{F}}^{2} \sigma_{\hat{D}}^{2}+\mu_{\hat{D}}^{2} \sigma_{\hat{F}}^{2}
$$

$\mu_{\hat{D}}$ is the expectation of $\hat{D}$, which for an unbiased estimator is equal to the actual difference, $D$, between the displacements of the two windows. $\sigma_{\hat{D}}^{2}$ is the variance of $\hat{D}$, which is approximately equal to the sum of the variances of the individual displacement estimates, $\hat{d}_{1}$ and $\hat{d}_{2}$ (it is exactly equal only if errors in $\hat{d}_{1}$ and $\hat{d}_{2}$ are uncorrelated, which is not the case for overlapping windows). $\mu_{\hat{F}}$ is the expectation of the reciprocal location spacing estimate, $\hat{F}$, which may correspond to the reciprocal of the spacing between consecutive windows. Finally, $\sigma_{\hat{F}}^{2}$ is the mean squared error between $\hat{F}$ and the actual reciprocal spacing, $F$. In general, $F$ is not equal to the reciprocal of the

${ }^{1} \mathrm{AMC}$ is the subject of UK patent application number GB 0606125.3. 


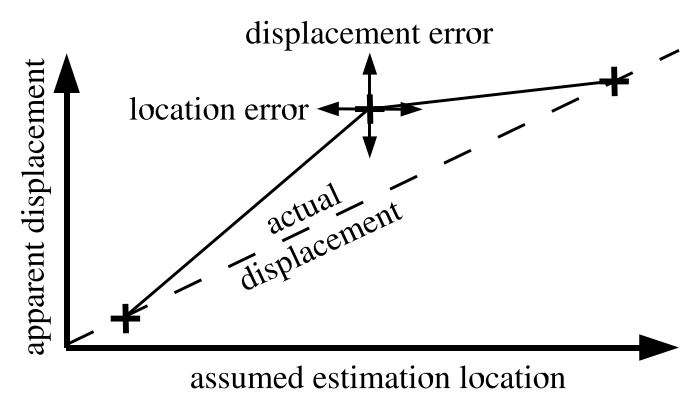

Figure 2: A practical estimate is displayed between two ideal estimates. There are two noise sources in practical displacement estimation.

window spacing, since the actual estimation locations, $\tau_{2}$ and $\tau_{1}$, do not generally correspond to the window centres.

We want to know what impact the terms in Equation 5 have on strain image quality. We consider a quality measure denoted $\mathrm{SNR}_{e}$, which has previously been defined $[4,37]$ and can be measured experimentally in images where the underlying strain field is known to be homogeneous.

$$
\mathrm{SNR}_{e}=\frac{\mu_{\hat{s}}}{\sigma_{\hat{s}}}
$$

$\mu_{\hat{s}}$ is the mean strain estimate and $\sigma_{\hat{s}}$ is the standard deviation. The presence of $\mu_{\hat{D}}^{2}$ in the third term of Equation 5 becomes important when $\mathrm{SNR}_{e}$ is evaluated. The noise contribution from the AM effect is therefore proportional to the strain, $s$, so the AM effect is expected to become the dominant source of strain estimation noise as the level of strain increases.

$$
\mathrm{SNR}_{e}=\left(\frac{\sigma_{\hat{D}}^{2} \sigma_{\hat{F}}^{2}+K_{1}^{2} \sigma_{\hat{D}}^{2}}{s^{2}}+K_{2}^{2} \sigma_{\hat{F}}^{2}\right)^{-\frac{1}{2}}
$$

Equation 7 is derived by substituting the RHS of Equation 5 into Equation 6 . The final result incorporates some simplifying assumptions. (1) $\mu_{\hat{s}}=s$. (2) The assumed value of $\hat{F}$ is usually a constant, i.e. $\mu_{\hat{F}}=\hat{F}=K_{1}$. (3) $\mu_{\hat{D}}=K_{2} s$ where $K_{2}$ is a constant (the expected shift equals the strain multiplied by the window spacing).

\subsection{Examples with pulse train signals}

Window matching tracks the displacement of the enclosed signal. However, if displacement varies within the window, then the actual signal displacement cannot be matched at all points. The location at which the actual displacement of the signal is equal to the displacement estimate varies depending on both signal and displacement field properties. In general, the estimation location comes from a random distribution throughout the window. It has low probability density at the ends, and in the absence of additional information its expectation is the window centre. Where the location cannot be estimated, it is best to assume that windows sample displacement at their centres. Unfortunately this means that the AM effect introduces displacement and strain estimation noise, as illustrated in Figures 1 and 2.

It is not possible to devise an estimator that both produces optimal displacement estimates and samples displacement at the centre of the window. This is because some portions of the signal may contain no information, or the quality of the information may be variable. This is demonstrated by examples with pulse train signals in Figure 3. In the absence of information between the pulses, an optimal displacement estimator tracks the displacement of the pulse(s) within each window. The example medium has been deformed by a uniform strain field, so displacement varies linearly with distance. The assumption of estimation at the window centre now leads to significantly different 


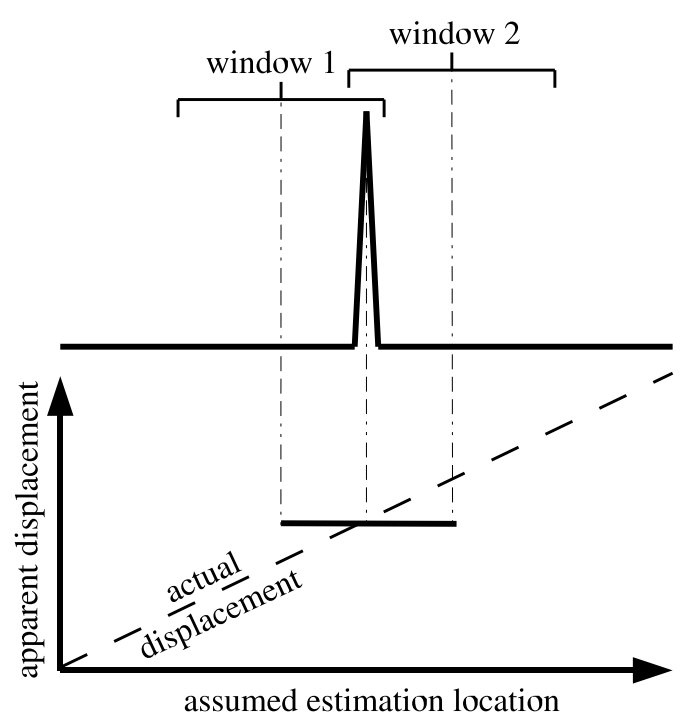

(a)

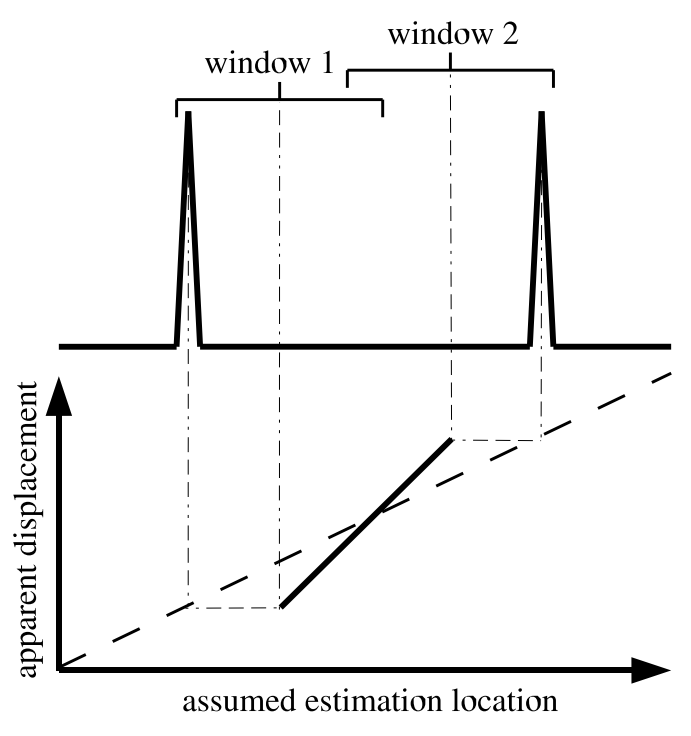

(b)

Figure 3: Extreme examples of the AM effect: (a) and (b) show the output of a perfect displacement estimator operating on different pulse train signals with uniform strain. The strain (displacement gradient) is (a) underestimated and (b) overestimated.

strain estimates if (a) overlapping windows track the same pulse, or (b) neighbouring windows track pulses at their extremities. When a uniform strain, $s$, is being tracked, and there is no displacement estimation error, the AM effect nonetheless distorts the result, such that the strain estimation lower bound is 0 for overlapping windows, and the upper bound is $s \times \frac{T+\Delta t}{\Delta t}$. $T$ is the window length and $\Delta t$ is the spacing between successive windows. For non-overlapping windows the lower bound is $s \times \frac{\Delta t-T}{\Delta t}$.

Of course, a real ultrasound signal is not a pulse train. Otherwise the AM effect could be corrected easily, by noting that displacement estimation occurs at the pulse locations. However, real ultrasound signals do incorporate amplitude variations, which are often large even over small distances. Lower amplitude sections usually have lower SNR, and a good displacement estimator should incorporate a mechanism for preferentially tracking the most reliable data. Ideally it should also be possible to estimate the actual displacement location when this is not equal to the window centre. The remainder of this section presents an analytical investigation of the AM effect in the context of some common ultrasonic strain estimation techniques.

\subsection{Phase-based methods}

We derive an approximate expression for the AM effect when windows are matched by identifying the zero crossing of the complex cross-correlation phase. Phase-based methods operate on analytic signals with real and imaginary parts, which are produced by applying the Hilbert transform (or some approximation thereof). The complex cross-correlation function and its phase may be expressed as follows.

$$
\begin{aligned}
\left\langle a_{1}, a_{2}\right\rangle(n \Delta t, \tilde{d}) & =\sum_{t=n \Delta t}^{n \Delta t+T} a_{1}^{*}(t) a_{2}(t+\tilde{d}) \\
\phi(n \Delta t, \tilde{d}) & =\angle\left\langle a_{1}, a_{2}\right\rangle(n \Delta t, \tilde{d})
\end{aligned}
$$

$a_{1}$ and $a_{2}$ are analytic ultrasound signals, ${ }^{*}$ denotes the complex conjugate, $n \Delta t$ is the location of the beginning of the analysis window in the pre-deformation signal, $T$ is the window length, 


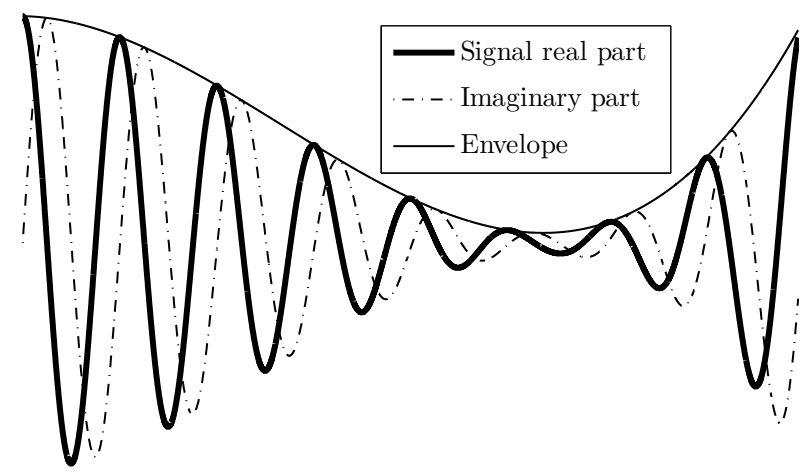

Figure 4: The signal model is a constant frequency sinusoid with arbitrary signal envelope, subject to an arbitrary deformation.

and $\tilde{d}$ is the candidate displacement applied to the post-deformation window to look for a match. Eventually the match or displacement estimate, $\hat{d}_{n}$, is found where $\phi$ has a zero crossing.

$$
\phi\left(n \Delta t, \hat{d}_{n}\right)=0
$$

It will be noted that if $\phi$ is only expressed in the range $[-\pi,+\pi]$ then a zero crossing occurs on average once for every wavelength shift in $\tilde{d}$. It is therefore necessary to incorporate a system for guiding the search to ensure that the correct zero crossing is always selected. This is analogous to eliminating "peak-hopping" errors from correlation coefficient analysis [41]. We do not investigate this issue here, but in practice we have found that it is always possible to eliminate this sort of outlier error by extending error detection and correction techniques similar to those described in past studies $[19,43]$.

To analyse the properties of phase-based methods, we use a simple signal model with no noise, where decorrelation occurs only as a result of the 1D signal stretching that accompanies mechanical strain. Our model of the pre-deformation signal, $a_{1}$, is a constant frequency sinusoid, scaled by a positive real signal envelope, $f$. This is illustrated in Figure 4.

$$
a_{1}(t)=f(t) e^{j \omega t}
$$

The main limitation of this model is the constant frequency assumption. Real RF ultrasound signals are narrowband, although the frequency may be substantially constant over short distances. In our model the post-deformation signal, $a_{2}$, is produced by an arbitrary temporal warping of $a_{1}$, such that every point, $a_{1}(t)$, undergoes a displacement, $d(t)$.

$$
a_{2}(t+d(t))=a_{1}(t)
$$

This is a simplification of the signal transformation that occurs in a real compression scan. Firstly, it will be noted that a uniform strain in our model gives rise to a change in the frequency centroid in the post-deformation signal, which will not usually be reflected in reality (although local frequency changes do occur). Secondly, we have assumed that the only change to the signal envelope will be a $1 \mathrm{D}$ warping. In reality, changes in the interference patterns of closely spaced scatterers introduce unpredictable components in the post-deformation signal, resembling the addition of an uncorrelated narrowband noise signal. Furthermore, axial compression in real materials with finite compressibility is inevitably accompanied by additional motions in the lateral and elevational directions. Nevertheless, we pursue analysis with our simplified model, and the predictions are later tested on real and simulated ultrasound data.

We examine the properties of the signals in matched windows. In general, the estimated displacement is similar but not equal to the local displacement at each position in the window. 
We therefore introduce a new symbol, $t_{2}$, denoting the pre-deformation location in $a_{1}$, of the data with which $a_{1}(t)$ is matched.

$$
t_{2}+d\left(t_{2}\right)=t+\hat{d}_{n}
$$

The complex cross-correlation function at the match is now expressed as follows.

$$
\begin{aligned}
\left\langle a_{1}, a_{2}\right\rangle\left(n \Delta t, \hat{d}_{n}\right) & =\sum_{t=n \Delta t}^{n \Delta t+T} a_{1}^{*}(t) a_{2}\left(t+\hat{d}_{n}\right) \\
& =\sum_{t=n \Delta t}^{n \Delta t+T} a_{1}^{*}(t) a_{1}\left(t_{2}\right) \\
& =\sum_{t=n \Delta t}^{n \Delta t+T} f(t) f\left(t_{2}\right) e^{j \omega\left(t_{2}-t\right)}
\end{aligned}
$$

In order to satisfy the match criterion (Equation 10), the imaginary part of the complex crosscorrelation function must be zero.

$$
\Im\left(\sum_{t=n \Delta t}^{n \Delta t+T} f(t) f\left(t_{2}\right) e^{j \omega\left(t_{2}-t\right)}\right)=0
$$

This leads to an alternative expression for the phase zero condition.

$$
\sum_{t=n \Delta t}^{n \Delta t+T} f(t) f\left(t_{2}\right) \sin \left(\omega\left(t_{2}-t\right)\right)=0
$$

It is noted that $t_{2}-t=\hat{d}_{n}-d\left(t_{2}\right)$ is the local discrepancy between the displacement estimate and its actual value. This is small, so $\omega\left(t_{2}-t\right) \ll \frac{\pi}{2}$ at all points in the window for typical window lengths and operating strains. It follows that Equation 18 may be simplified by applying the small angle approximation.

$$
\sum_{t=n \Delta t}^{n \Delta t+T} f(t) f\left(t_{2}\right) \omega\left(t_{2}-t\right) \simeq 0
$$

Equation 19 can be converted to an expression with clearer relevance to the physical deformation by examining the term $t_{2}-t$. This is performed as follows, employing the relation from Equation 13, and expanding a Maclaurin series about $d(t)$.

$$
\begin{aligned}
t_{2}-t & =\left\{\hat{d}_{n}-d(t)\right\}-\left\{d\left(t_{2}\right)-d(t)\right\} \\
& =\left\{\hat{d}_{n}-d(t)\right\}-\frac{\mathrm{d} d(t)}{\mathrm{d} t}\left\{t_{2}-t\right\}-\mathcal{O}\left\{\left(t_{2}-t\right)^{2}\right\} \\
& =\left\{\hat{d}_{n}-d(t)\right\}-s\left\{\hat{d}_{n}-d\left(t_{2}\right)\right\}-\mathcal{O}\left\{\left(\hat{d}_{n}-d\left(t_{2}\right)\right)^{2}\right\}
\end{aligned}
$$

Second order terms will be neglected, as will the term scaled by $s$ (strain), since the vast majority of previously documented ultrasonic strain imaging systems operate with $s \ll 1.0$. Now the result from Equation 22 is substituted into Equation 19.

$$
\sum_{t=n \Delta t}^{n \Delta t+T} f(t) f\left(t_{2}\right) \omega\left(\hat{d}_{n}-d(t)\right) \simeq 0
$$

Rearrangement yields a good approximate formula for the displacement estimate, $\hat{d}_{n}$.

$$
\hat{d}_{n} \simeq \frac{\sum_{t=n \Delta t}^{n \Delta t+T} f(t) f\left(t_{2}\right) d(t)}{\sum_{t=n \Delta t}^{n \Delta t+T} f(t) f\left(t_{2}\right)}
$$

We have shown that an approximation of the phase-based displacement estimate is a weighting of point displacements by the cross power of the local signal envelope. 


\subsection{Amplitude modulation correction}

We show that the actual estimation location can be estimated for the important case where strain may be considered constant at the scale of the individual windows. The constant strain condition is expressed mathematically as follows.

$$
d(t)=\alpha+s t
$$

We substitute this into Equation 24, and rearrange to produce a convenient form for the approximation.

$$
\begin{aligned}
\hat{d}_{n} & \simeq \frac{\sum_{t=n \Delta t}^{n \Delta t+T} f(t) f\left(t_{2}\right)(\alpha+s t)}{\sum_{t=n \Delta t}^{n \Delta t+T} f(t) f\left(t_{2}\right)} \\
& \simeq \alpha+\frac{s \sum_{t=n \Delta t}^{n \Delta t+T} f(t) f\left(t_{2}\right) t}{\sum_{t=n \Delta t}^{n \Delta t+T} f(t) f\left(t_{2}\right)}
\end{aligned}
$$

The location estimate, $\hat{\tau}_{n}$, is defined to be the position at which the displacement estimate approximation is equal to the actual displacement, i.e. $\hat{d}_{n} \simeq \alpha+s \hat{\tau}_{n}$. Hence,

$$
\hat{\tau}_{n}=\frac{\sum_{t=n \Delta t}^{n \Delta t+T} f(t) f\left(t_{2}\right) t}{\sum_{t=n \Delta t}^{n \Delta t+T} f(t) f\left(t_{2}\right)}
$$

These location estimates are substituted into Equation 1 to refine the strain estimates. This amplitude modulation correction (AMC) also allows a more accurate identification of the image region corresponding to the space between successive displacement estimates, thereby producing a more accurate correspondence between the physical locations of tissue features, and their apparent locations in strain or displacement images.

\subsection{Correlation coefficient methods}

Correlation coefficient methods have to date been the most popular approach for displacement tracking, at least within the ultrasonic strain imaging community. The correlation coefficient for real $\mathrm{RF}$ signals $r_{1}$ and $r_{2}$ at window $n$ with a candidate shift $\tilde{d}$ is evaluated as follows.

$$
\rho_{r_{1} r_{2}}(n \Delta t, \tilde{d})=\frac{\sum_{n \Delta t}^{n \Delta t+T} r_{1}(t) r_{2}(t+\tilde{d})}{\sqrt{\sum_{n \Delta t}^{n \Delta t+T} r_{1}(t)^{2} \sum_{n \Delta t}^{n \Delta t+T} r_{2}(t+\tilde{d})^{2}}}
$$

The displacement estimate is chosen to maximise the correlation coefficient.

$$
\hat{d}_{n}=\arg \max _{\tilde{d}} \rho_{r_{1} r_{2}}(n \Delta t, \tilde{d})
$$

In common with the analysis of phase-based methods, it would be highly desirable to derive a similar estimation location expression. The starting point is to identify the properties of stationary points (including the maximum) by differentiating $\rho_{r_{1} r_{2}}$ with respect to $\tilde{d}$. However, we have thus far been unable to derive an analytic expression for $\hat{\tau}_{n}$ in the case of correlation coefficient methods. Instead we apply the following heuristic, which is motivated by an assumption that the AM effect on correlation coefficient methods is similar to the effect on phase-based methods, for which AMC has already been derived.

$$
\hat{\tau}_{n}=\frac{\sum_{t=n \Delta t}^{n \Delta t+T}\left|r_{1}(t) r_{2}\left(t+\hat{d}_{n}\right)\right| t}{\sum_{t=n \Delta t}^{n \Delta t+T}\left|r_{1}(t) r_{2}\left(t+\hat{d}_{n}\right)\right|}
$$

Simulation results are included later to investigate whether or not this is a useful technique. 


\subsection{Benefits of amplitude modulation}

AMC increases the utility of displacement estimates from a spatially varying displacement field by estimating the actual estimation location. The alternative approach for handling the AM effect is to reduce the level of amplitude variation, for example by log compression of the signal envelope. This may be a useful technique in some circumstances, but it should be recognised that the AM effect may actually be beneficial for high quality displacement estimation.

Appendix A analyses a simple model of a generic displacement estimator, where short windows produce unreliable estimates, but the estimation variance can be reduced by using longer windows to take a weighted moving average. Following reasonable assumptions, it is shown that an optimal displacement estimator weights the importance of different signal sections in proportion with the local cross power, $r_{1}(t) r_{2}\left(t+\hat{d}_{n}\right)$. This outcome is similar in form to the approximation in Equation 24 for phase-based methods. It implies that the weighting becomes suboptimal if the amplitude is compressed, thereby reducing the accuracy of the displacement estimator. We therefore expect that if location estimation such as AMC is performed accurately, then the lowest strain estimation noise is achieved in the absence of log compression. It is less clear how far these conclusions apply to correlation coefficient methods, but the correlation coefficient also incorporates a weighting of some form, since high amplitude sections within a window have a greater impact on the overall correlation coefficient value.

\subsection{Adaptive strain estimators}

Adaptive strain estimators work on the principle of reversing the deformation that has occurred, to obtain the best match to the pre-deformation signal. Uniform strain is assumed at the scale of the individual windows - this is the same as the assumption applied in Section 2.3 to derive AMC. In 1D, an adaptive strain estimator uniformly stretches the post-deformation window until its similarity to the pre-deformation window is maximised. Past studies have shown that strain estimation error in these systems is lower than in conventional displacement-based methods. A feature of adaptive strain estimation is an increase in the correlation coefficient. This has been discussed by Srinivasan et al. [35], where increased correlation was identified as a cause of reduced strain estimation error. It is appropriate that in this paper we additionally note the likely contribution of the AM effect. If the local strain is actually uniform, adaptive strain estimation has the advantage of being able to correctly match the displacement at every point within the window. This means that for uniform strains the question of estimation location is irrelevant, because the correct displacement can be found everywhere. Tests of adaptive strain estimation on uniform strain simulations are therefore expected to be independent of the AM effect. It is for this reason that we employ an adaptive strain estimator as our AM suppression benchmark. It is also expected that the absence of the AM effect (by contrast with the other estimators) will result in a markedly different shape to the $\mathrm{SNR}_{e}$-strain characteristic.

\section{Experimental methods}

\subsection{Simulation}

Simulated RF ultrasound data has been generated using Field II [11]. The simulations have $2 \times 10^{5}$ scatterers positioned at random according to a uniform distribution throughout a $50 \times 50 \times 6 \mathrm{~mm}$ volume, with random scattering strengths distributed uniformly over the range $\left[0, \gamma_{\max }\right]$. The probe parameters model the 5-10 MHz probe of the Dynamic Imaging ${ }^{2}$ Diasus ultrasound machine, for which the point spread function has been measured experimentally — the pulse has a centre frequency of $6.0 \mathrm{MHz}$ and bandwidth $2.1 \mathrm{MHz}$ - and the sampling frequency is $66.7 \mathrm{MHz}$.

For each frame 128 A-lines have been simulated, spanning $40 \mathrm{~mm}$ in the lateral direction, recorded to a depth of $40 \mathrm{~mm}$. Simulations have been performed at a range of compressions $(0 \%$,

\footnotetext{
${ }^{2}$ http://www.dynamicimaging.co.uk
} 


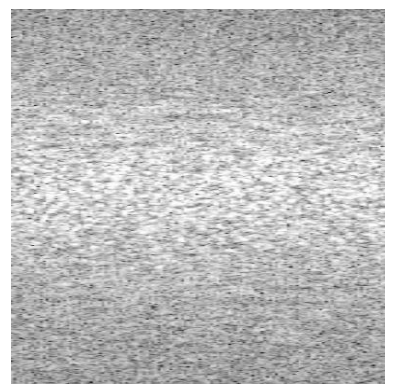

Figure 5: B-scan of simulated RF data.

$0.01 \%, 0.1 \% 0.5 \% 1.0 \%, 2.0 \%, 4.0 \%)$ by rescaling the axial spacing of the scatterers. This is important, because the relative performance of the strain estimation algorithms we compare is strain dependent. Five data sets have been generated for different scatterer fields. This contributes to the reliability of the results, which record the mean and standard deviation across the five data sets.

The Field II output has been converted to the RF ultrasound format of the Stradwin ${ }^{3}$ freehand 3D ultrasound system. RF samples are recorded with 16-bit signed integer precision. To ensure reproducibility of the resultant SNR and AM effects, the signals were normalised before conversion, such that in all cases the mean power is fixed at $V_{\mathrm{rms}}=2^{10}$, corresponding to a mean SNR of 71 $\mathrm{dB}$ in the presence of quantisation noise. Tests have also been performed on simulated data with additive white Gaussian noise, reducing the SNR to $20 \mathrm{~dB}$. Figure 5 shows an example B-scan from the simulated data.

\section{$3.2 \quad$ In vitro and in vivo scanning}

Scans have been performed using a Dynamic Imaging Diasus ultrasound machine with a 5-10 MHz probe, sampled at $66.7 \mathrm{MHz}$ by a Gage ${ }^{4}$ CompuScope 14200 analogue-to-digital converter, with a PC running the Stradwin freehand 3D ultrasound software. As per previous work [19], frames were acquired at $30 \mathrm{~Hz}$ during a freehand scan, and exaggerated palpating movements were not necessary. The images are used only for qualitative assessment of the strain estimation algorithms. Results are shown for (1) olive/gelatin phantom mimicking a stiff inclusion in soft tissue, (2) tissue-mimicking phantom with two layers, (3) human male breast in vivo, (4) human calf muscle in vivo.

\subsection{Strain estimators}

For comparative purposes, we test phase, correlation coefficient and adaptive strain estimators. The performance of phase and correlation coefficient estimators is compared for several variations: uncorrected strain estimation, log compression, limit log compression and AMC. Quantitative tests use simulation data, where the performance is measured by evaluating $\mathrm{SNR}_{e}$; the strain standard deviation is calculated from the raw strain estimates, where no smoothing has been applied. For a qualitative assessment, we also present example images from in vitro and in vivo scans.

Fair comparison is made possible by fixing the window parameters across all of the estimators in each test. It should be noted, that where there is a priori knowledge of a uniform strain field, the process of imaging strain by differencing closely spaced windows serves only to introduce noise; instead, windows separated by a large distance should be differenced in order to achieve an $\mathrm{SNR}_{e}$ that becomes arbitrarily high for large window spacing. Alternatively, in practical systems it is sensible to match larger numbers of closely spaced windows, and to combine their estimates

\footnotetext{
${ }^{3}$ http://mi.eng.cam.ac.uk/ ${ }^{\text {rwp/stradwin/ }}$

${ }^{4}$ http://www.gage-applied.com
} 
by filtering methods such as least squares or wavelet decomposition. To varying degrees, these techniques reduce both noise and resolution, although the AM effect will remain important. Since the entire purpose of our study is to investigate the noise that is introduced by erroneous estimation location assumptions, and to evaluate the performance of the proposed AMC technique, in our quantitative tests we stick to the method of differencing windows at a fixed window-spacing, $\Delta t=$ $2.7 \lambda$ (i.e. $0.45 \mu \mathrm{s}, 0.35 \mathrm{~mm}, 30 \mathrm{RF}$ samples at $66.7 \mathrm{MHz}$ ). The window length, $T$, is varied between tests, with the chosen length stated in each case.

The remainder of the experimental methods section provides a full description of each estimator, the properties of the simulation data, and the nature of the in vitro and in vivo ultrasound scans.

\subsubsection{Efficient phase zero search}

The efficient phase zero search (EPZS) is drawn from previous work [19] adapting the concept of Pesavento et al. [29]. To summarise, a 5-10 MHz filter is applied to the RF data $\left(r_{1}, r_{2}\right)$ before converting to analytic signal representations $\left(a_{1}, a_{2}\right)$, which are modulated to the baseband $\left(a_{b 1}\right.$, $\left.a_{b 2}\right)$ to enhance the accuracy of linear interpolation. $a_{b 2}$ must be estimated at subsample locations by baseband linear interpolation, to enable accurate subsample estimation of $d$ (for a discussion of interpolation frequency responses, see Proakis and Manolakis [30]). Phase-based methods require that the displacement of the analysis window is known already to within $\lambda / 2$; this is achieved by initialising each window with the final displacement estimate from the preceding one; windows at the top of each A-line are initialised with $\tilde{d}=0$. Displacement estimates are differenced to produce strain estimates following Equation 1.

The estimation location is usually assumed to be the window centre.

$$
\hat{\tau}_{n}=n \Delta t+\frac{T}{2}
$$

The phase is preserved but the amplitude is partially suppressed when the signal is log compressed according to the following formula.

$$
a_{b, \log }(t)=\log \left(1+c\left|a_{b}(t)\right|\right) e^{j \arg a_{b}(t)}
$$

$c$ is the compression factor. The larger the value of $c$, the smaller the amount of amplitude information that is retained, since the size of variations in the log compressed amplitude becomes smaller compared to the mean value. We refer to this algorithm as EPZSL1. As $c \rightarrow \infty$ all of the amplitude information is discarded, since log compressed amplitude variations become infinitely smaller than the mean. Limit log compression has a simpler form.

$$
a_{b, \log }(t)=e^{j \arg a_{b}(t)}
$$

We refer to limit log compression as EPZS_L2. For phase-based methods, EPZS_L2 is the counterpart of one-bit compression or zero crossing techniques in correlation coefficient methods [4, 34, 39]. We also present results for EPZS with AMC, referred to as EPZS_A. In addition to producing analytic signals, we detect the signal envelope, $|a|$, which is exploited as follows for AMC estimation of $\hat{\tau}_{n}$ (c.f. Equation 28).

$$
\hat{\tau}_{n}=\frac{\sum_{t=n \Delta t}^{n \Delta t+T}\left|a_{1}(t)\right|\left|a_{2}\left(t+\hat{d}_{n}\right)\right| t}{\sum_{t=n \Delta t}^{n \Delta t+T}\left|a_{1}(t)\right|\left|a_{2}\left(t+\hat{d}_{n}\right)\right|}
$$

EPZS_L2 uses none of the amplitude information, so the AMC version of $\hat{\tau}_{n}$ is identical to the window centre assumption. However, EPZS_L1 still exhibits a degree of AM susceptibility, so results are presented for an algorithm combining EPZS_L1 with AMC (operating on the log compressed signal envelope), referred to as EPZS_LA.

\subsubsection{Correlation coefficient maximiser}

The correlation coefficient maximiser (CCM) searches initially at integer sample locations for the maximum value of the cross correlation coefficient (see Equation 29). The estimate is refined by 
allowing subsample values of $d$ and interpolating $r_{2}$ at subsample locations. Again, a complex baseband representation of $r_{2}$ allows highly accurate subsample interpolation, as with EPZS, but in CCM it is converted back to a subsample real signal for the correlation coefficient calculation. This requires the following calculation, where $\omega_{m}$ is the modulation frequency that was used earlier to shift the analytic signal down to the baseband.

$$
r_{2}(t)=\Re\left\{a_{b 2}(t) e^{j \omega_{m} t}\right\}
$$

$\hat{\tau}$ is again usually assumed to be the window centre (Equation 32). Log compression (CCM_L1) is tested as a means of reducing the error in $\hat{\tau}$, using the following formula, as first proposed by Céspedes and Ophir [4].

$$
r_{\log }(t)=\log (1+c|r(t)|) \operatorname{sign}(r(t))
$$

To maximise algorithm performance, the full RF signal is used for subsample interpolation of $r_{2}$, which is only log compressed at the moment of computing the correlation coefficient. In the limiting case as $c \rightarrow \infty$ variations in the log compressed signal magnitude become infinitely smaller than the mean magnitude, so only the sign is important. A simpler expression may be used.

$$
r_{\log }(t)= \begin{cases}+1 & r(t) \geq 0 \\ -1 & r(t)<0\end{cases}
$$

Subsample interpolation actually still employs the full RF signal, so zero crossings are identified with high accuracy. We call this variation CCM L2. It has previously been described as one-bit compression [4] and is equivalent to zero crossing methods [34]. AMC is applied to CCM following Equation 31, which is referred to as CCM_A. AMC is also applied alongside non-limiting log compression in CCM_LA.

\subsubsection{Adaptive strain estimator}

Typical adaptive strain estimators from previous studies have two search dimensions - displacement and stretch - for each spatial dimension of strain estimation [1,33]. A typical algorithm has the following stages: (1) each post-deformation window is shifted till the best match is located; (2) the shifted window is stretched to maximise a similarity measure; (3) displacement is re-estimated for the stretched window; and (4) the process repeats iteratively until convergence. Once arrays of displacement and stretch have been calculated, either the displacement estimates may be differenced (as in displacement-based methods) to re-estimate strain, or the stretch estimates may be displayed directly (which is the approach followed in this study). An estimator of this form was observed by the authors to produce significantly better strain images than those that are achieved by the basic displacement estimation approaches, with the greatest improvement for high strains. SAD was found to outperform the correlation coefficient, so this is the chosen signal similarity measure. The origin of this difference may lie in the fact that often $\rho_{r_{1} r_{2}} \simeq 1.0$ at the correct stretch, in which case SAD is less prone to quantisation errors.

It has subsequently been noted that a minor modification to the adaptive stretching algorithm yields a further performance improvement. The modification concerns the way that displacement is estimated: our adaptive strain estimator (ASE) estimates the locations of the windows directly from the strain estimates, rather than searching over two dimensions. This has been found to yield higher $\mathrm{SNR}_{e}$.

The initialisation of EPZS depends on the fact that the displacement at the top of each A-line is zero. Similarly, ASE searches only over strain (and not over displacement) in the top window of each A-line. This utilises the prior knowledge that a search over displacement could only degrade the accuracy of the estimate in the event that a non-zero displacement were found for the top of the window. The displacement at subsequent windows is estimated accurately by integrating the estimated strains, where it will be recognised that integration is a noise-suppressing operation. The offset of the first sample in a succeeding overlapping window is, of course, not equal to the displacement at the end of the first window. Rather, the relationship we assume is illustrated in 


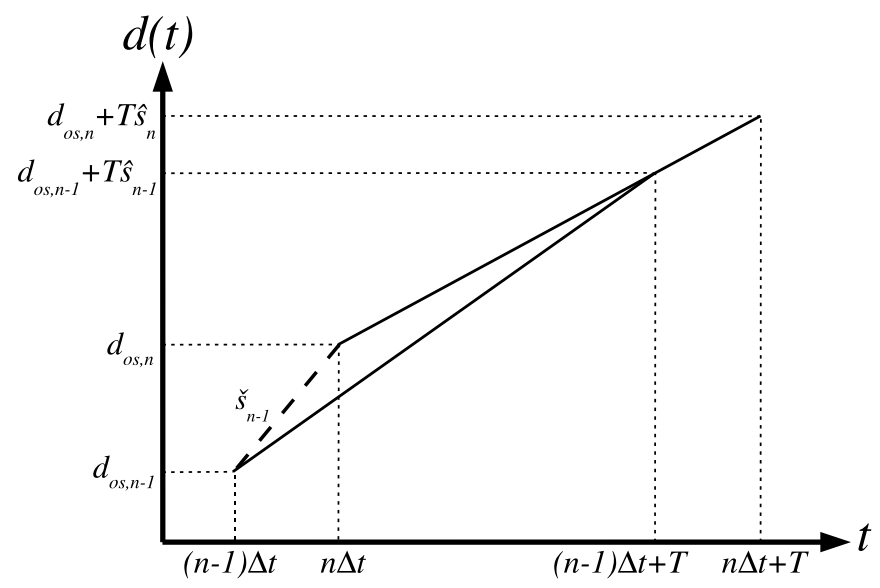

Figure 6: Illustration displacement offsets in ASE. The estimated displacement of window $n$ is fixed at the end displacement of window $n-1$, while $\hat{s}_{n}$ is the estimated displacement gradient across the window. This yields $\check{s}_{n-1}$ as the higher resolution strain estimate for the previous window.

Figure 6, where estimated strains are displayed as gradients on a plot of displacement against time. The window strain estimate multiplied by the window length, $T \hat{s}_{n-1}$, provides the best estimate for the displacement difference between the end and the start. The following window is therefore pinned at this end point, and stretched on either side to find the next estimate, $\hat{s}_{n}$. This means that the offset displacement at the start of window $n$ depends on: the offset of window $n-1$, the previous window stretch, and the candidate window stretch, $\tilde{s}$.

$$
d_{o s, n}=d_{o s, n-1}+\hat{s}_{n-1} T-\tilde{s}(T-\Delta t)
$$

This leads immediately to a second result for increased resolution with overlapping windows. An estimate that resolves strain changes at the scale of the shift between windows (thereby matching the resolution of the displacement methods) is produced as follows.

$$
\check{s}_{n-1}=\left\{\hat{s}_{n-1} T-\hat{s}_{n}(T-\Delta t)\right\} / \Delta t
$$

This is a consequence of the geometry in Figure 6. Increased resolution comes at a cost of increased estimation noise. We present results using $\check{s}$ rather than $\hat{s}$, however, since the higher resolution of $\check{s}$ makes it the appropriate comparison with the displacement-based methods. Having dealt with the displacement offset and resolution issues, the basic form of the ASE search is an optimisation problem similar to the other methods.

$$
\operatorname{SAD}(n, \tilde{s})=\sum_{t=n \Delta t}^{n \Delta t+T}\left|r_{1}(t)-r_{2}\left(t+d_{o s, n}+\tilde{s}\left(t+\frac{1}{2 f_{s}}-n \Delta t\right)\right)\right|
$$

$f_{s}$ is the sampling frequency. $\hat{s}_{n}$ minimises $\operatorname{SAD}(n, \tilde{s})$.

$$
\hat{s}_{n}=\arg \min _{\tilde{s}} \operatorname{SAD}(n, \tilde{s})
$$

It might be possible to adapt fast algorithms to this optimisation problem, but for now we use an exhaustive search.

\section{Results}

Quantitative results indicate the advantages and disadvantages of each technique. Important trends are illustrated by graphs. Where there is space for error bars these extend to one standard deviation either side of the mean. We also present strain images for qualitative assessment. 


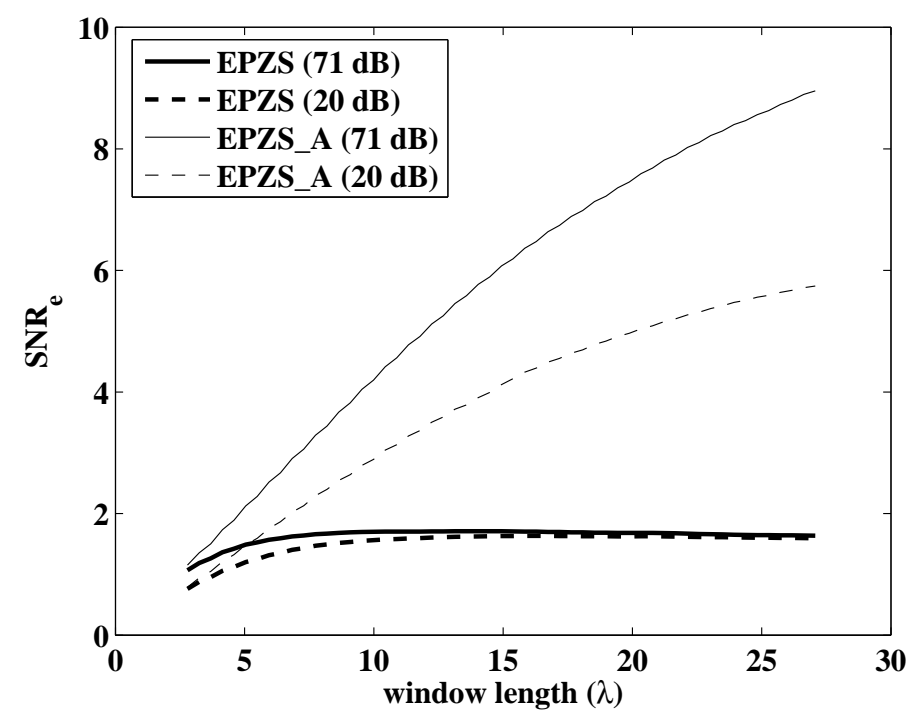

Figure 7: $\mathrm{SNR}_{e}$ against window length for EPZS and EPZS_A, with both $71 \mathrm{~dB}$ and $20 \mathrm{~dB}$ data at $0.5 \%$ strain. Uncorrected EPZS with $71 \mathrm{~dB}$ data reaches a plateau at $T=10 \lambda$, which the 20 $\mathrm{dB}$ results converge towards for long windows. When $\mathrm{AMC}$ is applied there is no such plateau and much higher $\mathrm{SNR}_{e}$ is achieved - $\mathrm{SNR}_{e}$ is initially a linear function of window length, and it continues to increase for long windows, although the gradient becomes less steep.

\subsection{Window length}

Results for EPZS, EPZS_A, CCM, CCM_A and ASE with window lengths, $T$, in the range 2.8$27.1 \lambda$ indicate a suitable choice of $T$ for the later tests. They also serve as a first opportunity for assessing the AMC technique. Figures 7 and 8 show performance against window length at $0.5 \%$ strain, while Figure 9 shows the effect of window length on EPZS_A and ASE at a higher strain. $13.5 \lambda$ is employed for all other results in this report.

To illustrate the practical meaning of $\mathrm{SNR}_{e}$, Figure 10 shows strain images at $0.5 \%$ compression. The characteristics of the images can be compared with the corresponding $\mathrm{SNR}_{e}$ results from the graphs. The images have a linear scale with 0 (black) representing zero strain, 127.5 (mid-grey) is the simulated strain of $0.5 \%$ and 255 (white) represents $1 \%$. Saturation occurs at 0 and 255 , and no smoothing has been applied, so each section between successive estimation locations has constant brightness. An ideal estimator would yield a uniform greyscale level, but this is unachievable in practice.

\subsection{Compression factor}

A justification is presented for the choice of log compression factor in the algorithms EPZS_L1, EPZS_LA, CCM_L1 and CCM_LA. The effect of log compression varies to a large degree depending on the strain level, so Figures 11-13 show results at strains representing the smallest, largest, and mid-range in the simulation data. It is evident that log compression is not always desirable, but the choice of $c$ reflects a value that is likely to boost $\mathrm{SNR}_{e}$ in high strain regions, whilst avoiding extreme degradation of low strain estimates. $c=10^{3}$ is employed for all of the remaining results. 


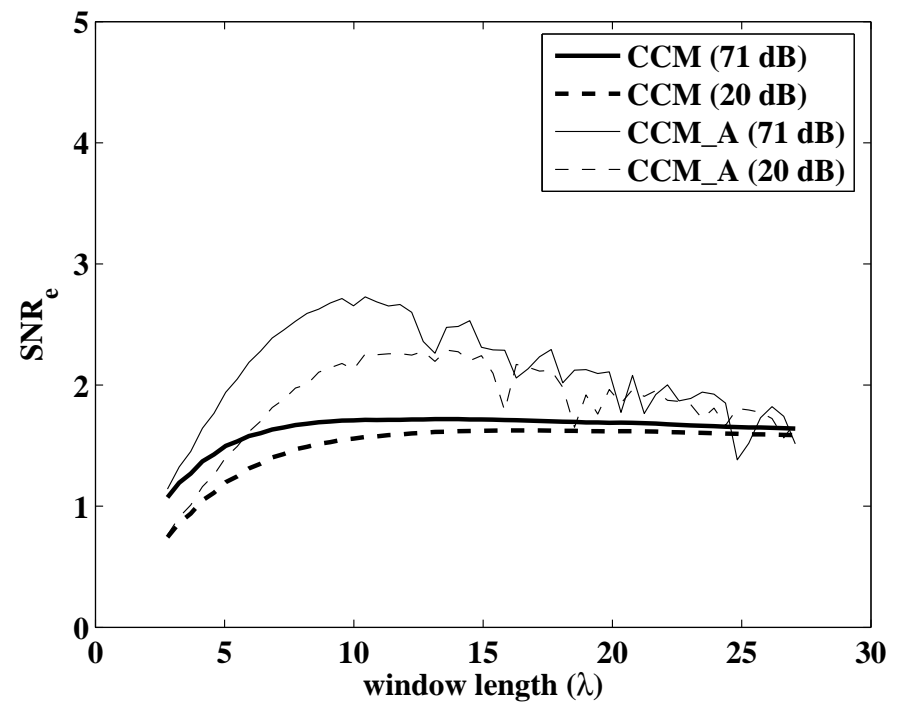

Figure 8: $\mathrm{SNR}_{e}$ against window length for $\mathrm{CCM}$ and $\mathrm{CCM} \_$, with both $71 \mathrm{~dB}$ and $20 \mathrm{~dB}$ data at $0.5 \%$ strain. Uncorrected CCM is almost identical to EPZS. However, AMC is obviously less accurate for CCM, since the improvement with CCM_A is much smaller and the results are erratic for long windows.

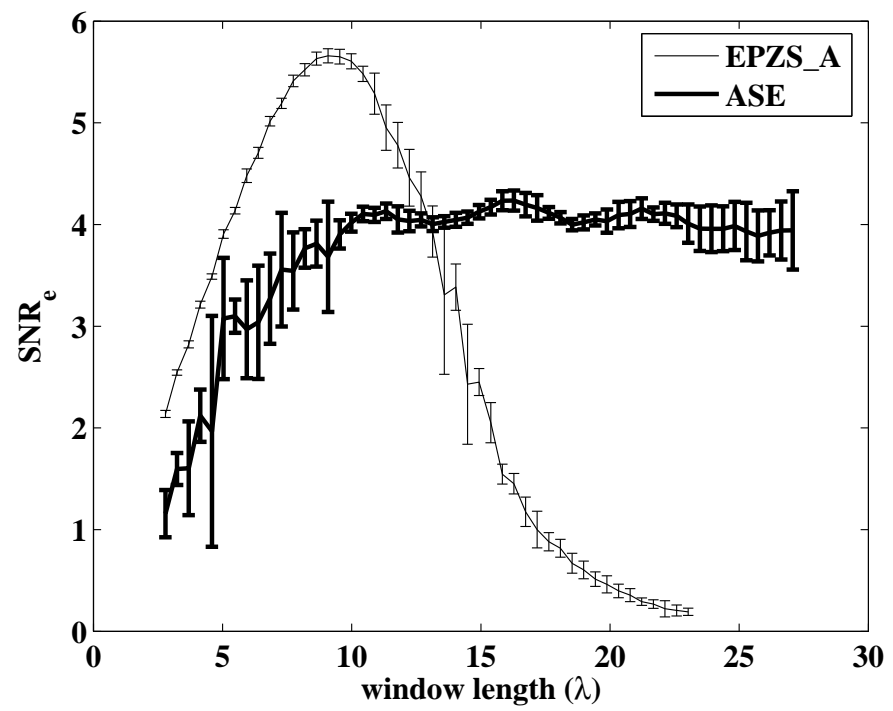

Figure 9: $\mathrm{SNR}_{e}$ against window length for EPZS_A and ASE, with $20 \mathrm{~dB}$ data at $4 \%$ strain. ASE performs less well with short windows, but it reaches a high and fairly constant level of performance for $T>10 \lambda$. EPZS_A, by contrast, performs well with short windows and has a higher peak $\mathrm{SNR}_{e}$. However, windows with $T>10 \lambda$ have a differential displacement of $>0.4 \lambda$ between the ends, so in this range EPZS_A suffers substantially increased decorrelation and estimation noise. 


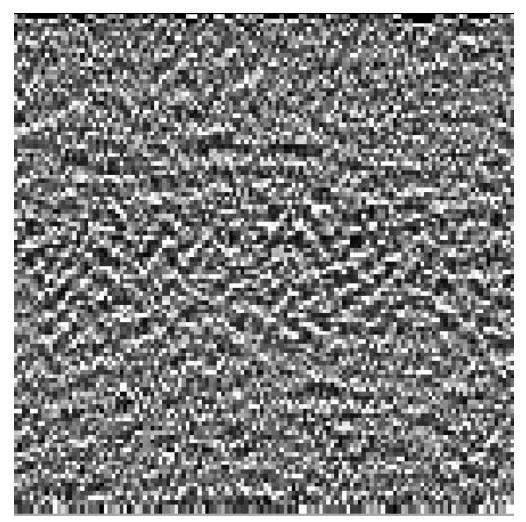

(a) $\mathrm{SNR}_{e}=1.63$

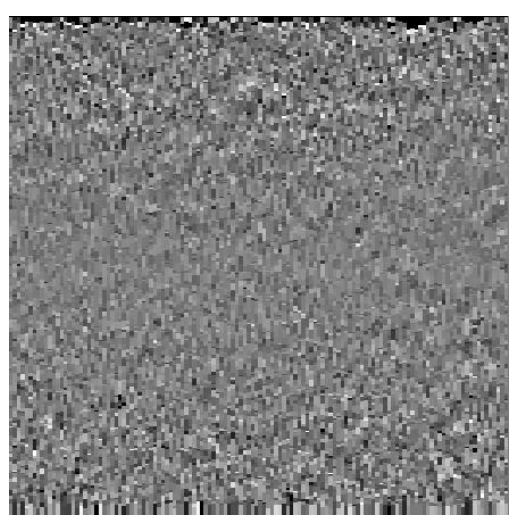

(c) $\mathrm{SNR}_{e}=3.86$

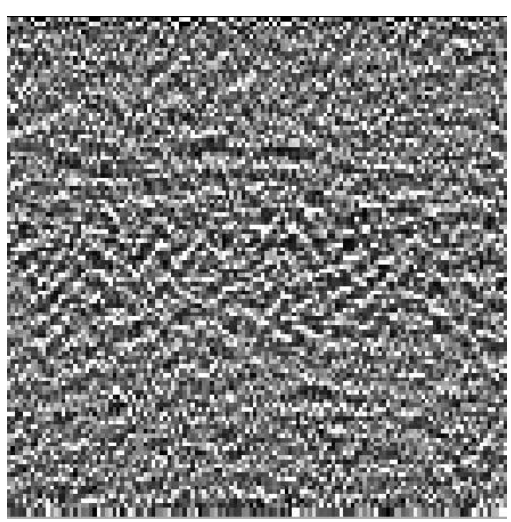

(b) $\mathrm{SNR}_{e}=1.62$

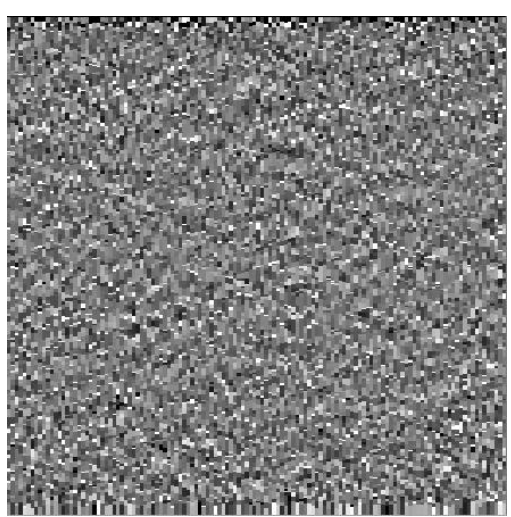

(d) $\mathrm{SNR}_{e}=2.05$

Figure 10: Strain images for a $0.5 \%$ compression with $20 \mathrm{~dB}$ data using $T=13.5 \lambda$ : (a) EPZS; (b) CCM; (c) EPZS_A; (d) CCM_A. The performance of EPZS and CCM is similar, though EPZS_A performs considerably better than CCM_A. 


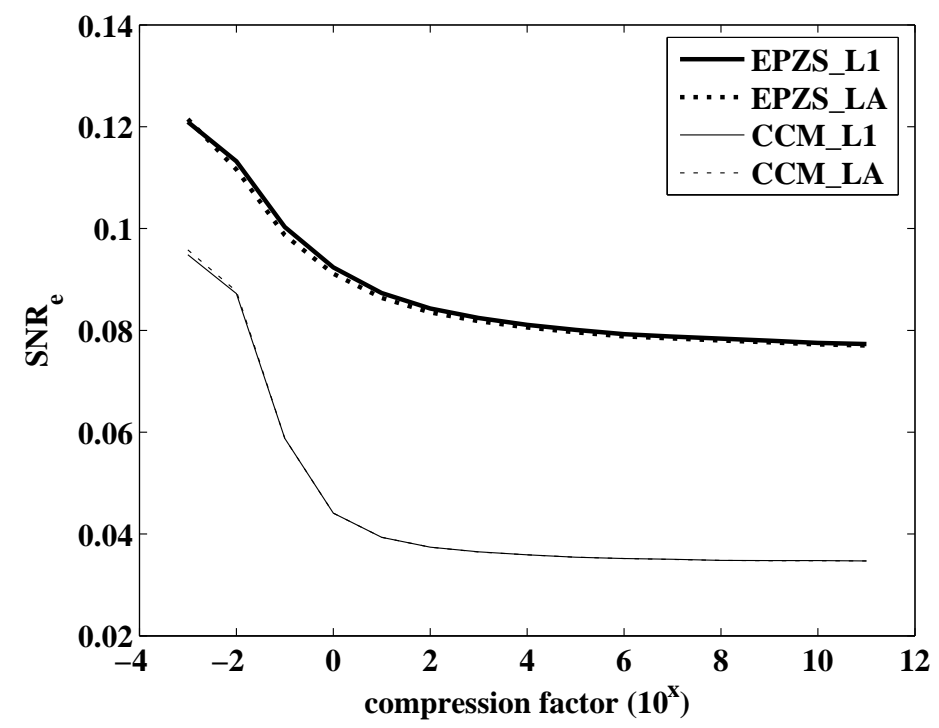

Figure 11: SNR $e_{e}$ results for EPZS_L1, EPZS_LA, CCM_L1 and CCM_LA with 20 dB data at $0.01 \%$ strain as a function of $c$, the compression factor. At low strains, the main effect of log compression is increased noise. This effect is more pronounced with CCM_L1. AMC has almost no effect in these images.

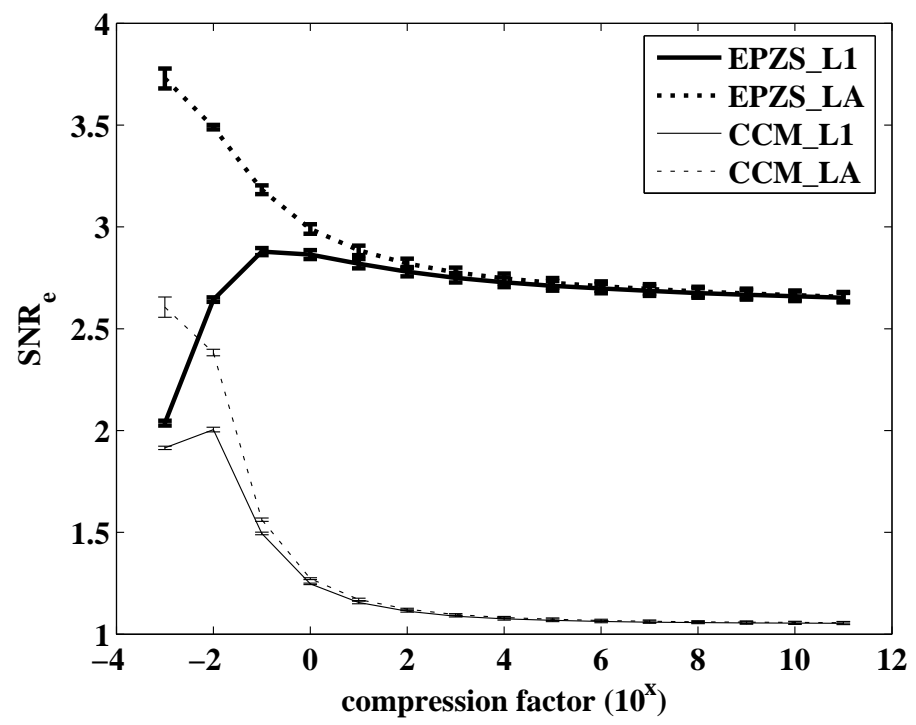

Figure 12: SNR $_{e}$ results for EPZS_L1, EPZS_LA, CCML1 and CCMLA with 20 dB data at $0.5 \%$ strain as a function of $c$, the compression factor. At this strain, $\log$ compression significantly improves the performance of EPZS_L1. CCM_L1 is also improved by slight log compression. Better performance is produced by AMC, although this is degraded by log compression, so as $c \rightarrow \infty$ EPZS_LA and CCM_LA converge with the curves where AMC has not been applied. 


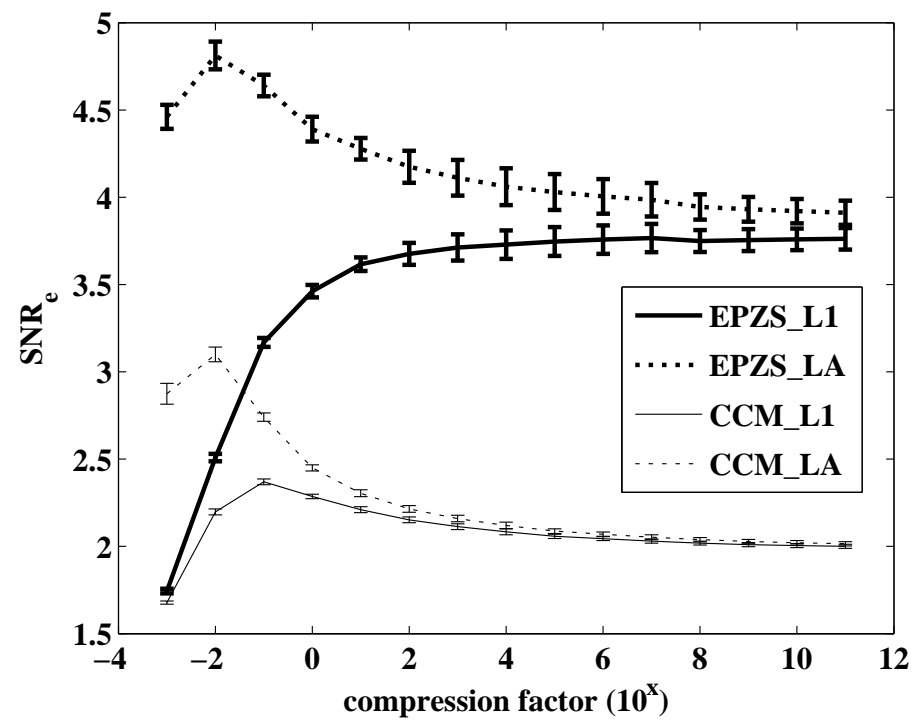

Figure 13: SNR $_{e}$ results for EPZS_L1, EPZS_LA, CCM_L1 and CCM_LA with 20 dB data at $4 \%$ strain as a function of $c$, the compression factor. At this strain all of the algorithms can be improved by applying an appropriate level of log compression. The greatest improvement is exhibited by EPZS_L1, while the ACM algorithms are still degraded by high compression factors, and they eventually converge with the curves where AMC has not been applied.

\subsection{Strain dependence}

With parameters $T$ and $c$ selected as per the preceding sections, Figures 14-16 compare the performance of EPZS, EPZS_L1, EPZS_L2, EPZS_LA, EPZS_A, CCM, CCM_L1, CCM_L2, CCM_LA, CCM_A and ASE across a range of strains.

\subsection{In vitro and in vivo results}

Finally, images from real ultrasound scans are presented. For the sake of concision, we restrict ourselves to EPZS, EPZS_L1, EPZS_L2 and EPZS_A, allowing a qualitative assessment of log compression and AMC when applied to real data. The images in Figures 17-20 have been smoothed slightly by estimating strain with a $1 \mathrm{~mm}$ least squares filter along the axial direction; no other filtering has been applied and the values of parameters $T$ and $c$ are unchanged.

\section{Discussion}

\subsection{Interpretation of results}

Window length results in Figure 7 show that AMC is extremely effective when applied to EPZS, which validates the analysis in Sections 2.2 and 2.3. Notice that while increasing the window length is known to reduce $\sigma_{\hat{D}}^{2}$, nevertheless the uncorrected algorithm quickly reaches a plateau: this is because the primary source of noise is the AM effect when long windows are used. Meanwhile, when AMC is applied the remaining noise is mainly due to $\sigma_{\hat{D}}^{2}$, so higher $\mathrm{SNR}_{e}$ is achieved with the $71 \mathrm{~dB}$ data. It is encouraging, however, that the curve for $20 \mathrm{~dB}$ data has the same form as for $71 \mathrm{~dB}$ data. This shows that although AMC was derived considering noiseless data, the technique has a similar effect in the presence of noise. 


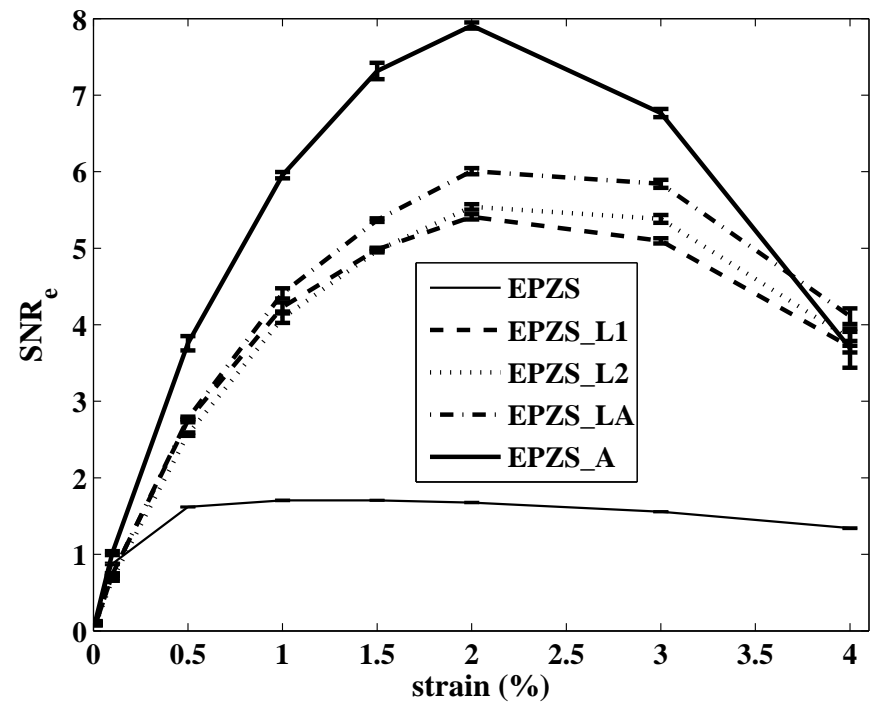

Figure 14: $\mathrm{SNR}_{e}$-strain characteristics for the EPZS family of algorithms with 20 dB data. EPZS_A has the best performance across a wide range of strains, although the $\mathrm{SNR}_{e}$ is lower at high strains and at $4 \%$ the best performance is from EPZS_LA.

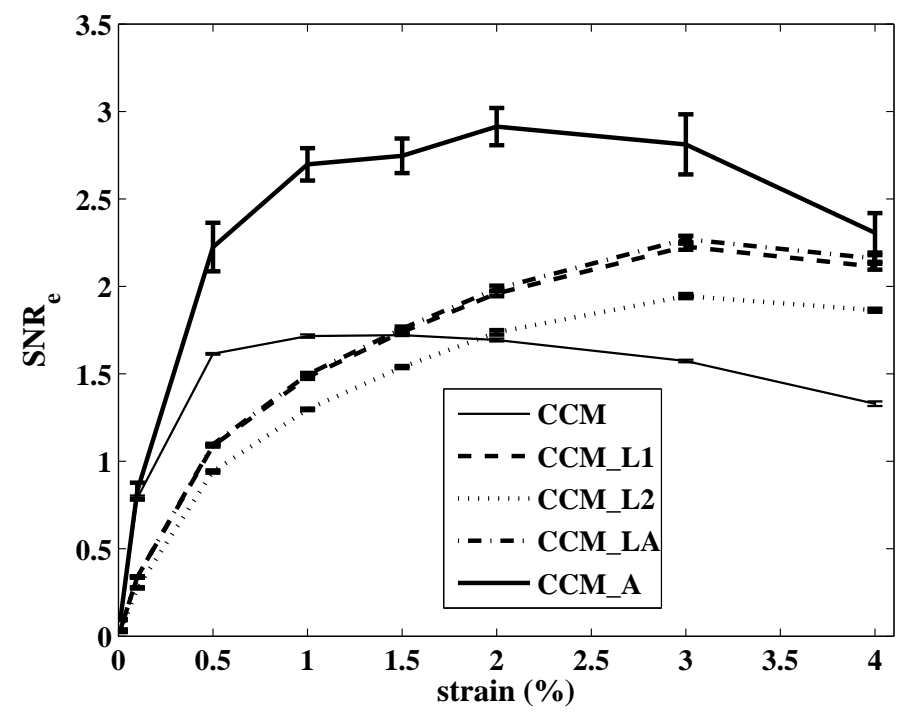

Figure 15: $\mathrm{SNR}_{e}$-strain characteristics for the CCM family of algorithms with $20 \mathrm{~dB}$ data. At all strains CCM_A significantly outperforms the other algorithms. In the absence of AMC, log compression boosts CCM performance at strains above 1.5\%, though the best log compression performance comes from the combination algorithm, CCM_LA. 


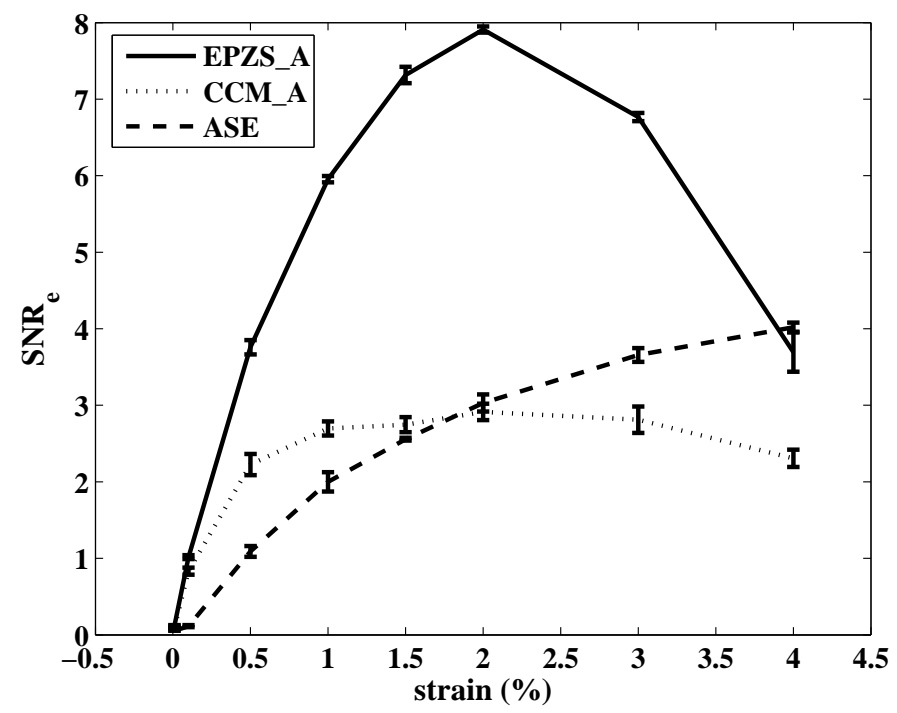

Figure 16: $\mathrm{SNR}_{e}$-strain characteristics for EPZS_A, CCM_A and ASE with 20 dB data. These are the best algorithms from each of the three families. EPZS_A performs best across most strains, though ASE does slightly better at 4\%, where the other algorithms have lower $\mathrm{SNR}_{e}$ owing to significant decorrelation.

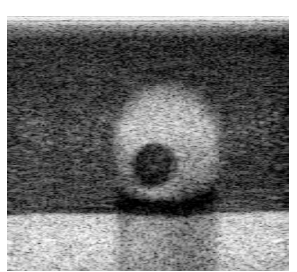

(a)

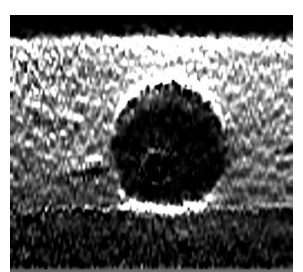

(b)

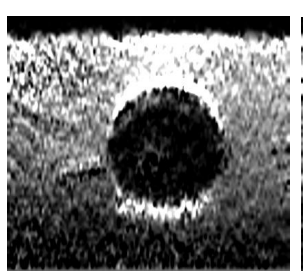

(c)

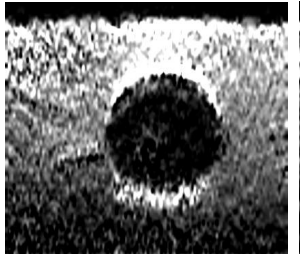

(d)

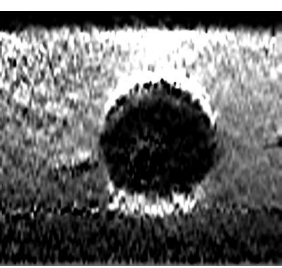

(e)

Figure 17: Olive/gelatin phantom: (a) B-scan, (b) EPZS (white=255=1\% strain), (c) EPZSL1, (d) EPZS_L2, (e) EPZS_A.

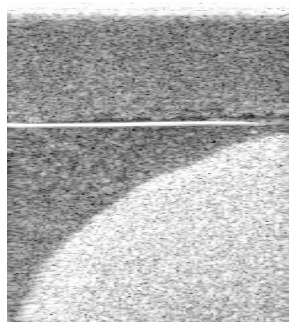

(a)

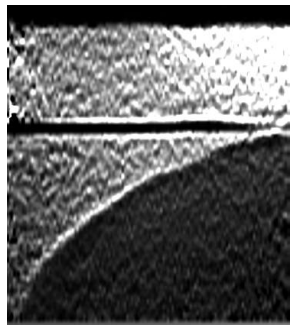

(b)

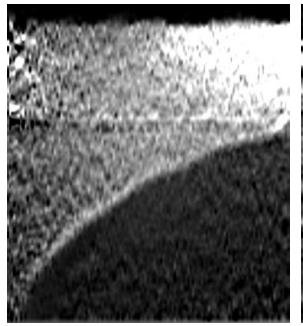

(c)

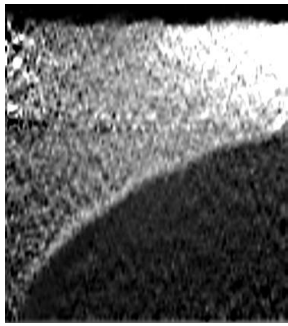

(d)

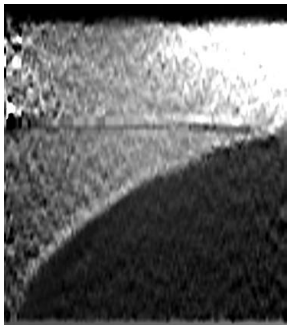

(e)

Figure 18: Gelatin phantom with two regions: (a) B-scan, (b) EPZS (255=0.8\%), (c) EPZS_L1, (d) EPZS_L2, (e) EPZS_A. 


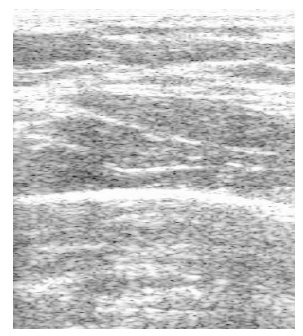

(a)

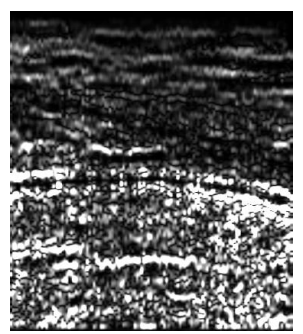

(b)

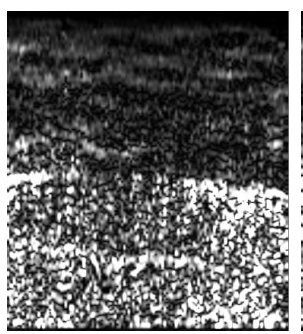

(c)

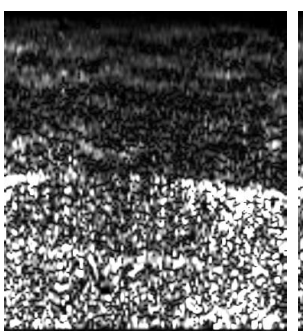

(d)

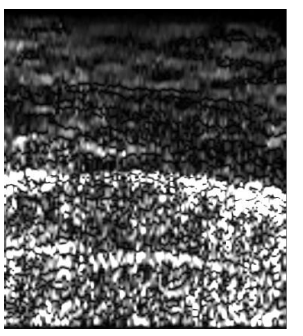

(e)

Figure 19: Human male breast: (a) B-scan, (b) EPZS (255=0.8\%), (c) EPZS_L1, (d) EPZS_L2, (e) EPZS_A.

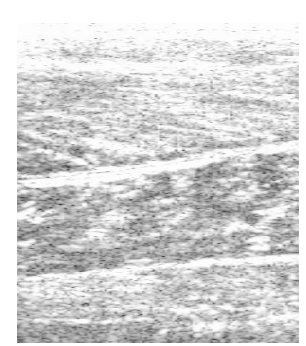

(a)

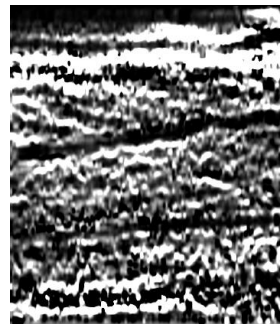

(b)

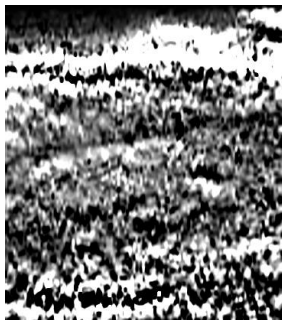

(c)

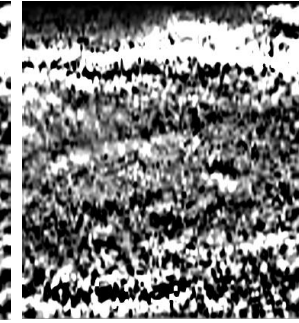

(d)

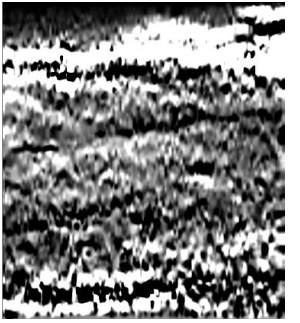

(e)

Figure 20: Human male calf: (a) B-scan, (b) EPZS (255=0.8\%), (c) EPZS_L1, (d) EPZS_L2, (e) EPZS_A.

Note from Figure 8 that the performance of uncorrected CCM is almost identical to EPZS. However, AMC for CCM is less successful, which probably reflects the lack of a formal derivation, rather than implying that it is not possible to correct the AM effect in this case. The formula in Equation 31 was based on intuition. The derivation of a superior CCM_A algorithm would be a valuable contribution, since it is a considerably more challenging mathematical problem than EPZS_A.

Figure 9 confirms that ASE offers an alternative route to high-performance strain estimation. In particular, it is possible to achieve good performance using arbitrarily long windows. This means that locations of extremely high strain will not be subject to reduced $\mathrm{SNR}_{e}$ when the window length has been chosen for optimal performance at a range of lower expected strains. It is also interesting to note that EPZS_A actually outperforms ASE for short window lengths, and EPZS_A has the higher peak performance. Of course, EPZS_A performs less well with longer windows, where high strains cause significant decorrelation. The window length chosen for subsequent tests $(T=13.5 \lambda)$ was determined by two factors: (1) long windows eventually reduce resolution in practical strain imaging; and (2) $13.5 \lambda$ is a sensible balance for near-optimal performance across all algorithms at all strains in the range $0.01-4 \%$.

The log compression results in Figures 11-13 demonstrate the behaviour that was predicted in Section 2.5. $\sigma_{\hat{D}}^{2}$ dominates at the low strain in Figure 11, so the key to noise suppression is using all of the amplitude data to maximise the accuracy of the displacement estimates. Therefore, log compression serves only to degrade performance. EPZS and EPZS_A have identical $\sigma_{\hat{D}}^{2}$, while $\sigma_{\hat{F}}^{2}$ is less important, so AMC is irrelevant. The same observation applies to CCM and CCM_A. However, EPZS and EPZS_A are degraded less severely by log compression, since the retention of phase information makes these algorithms more robust. CCM only uses the real signal, so $\sigma_{\hat{D}}^{2}$ increases rapidly with log compression as information is discarded.

However, $0.5 \%$ strain in Figure 12 is already sufficiently high for the noise contribution of $\sigma_{\hat{F}}^{2}$ to become important. Log compression yields a significant improvement in EPZS_L1, and slight 
log compression also improves CCM_L1. Better performance is achieved by the AM corrected algorithms, although these are still degraded by log compression. EPZS_A and CCM_A eventually converge with the uncorrected curves as $c \rightarrow \infty$. Log compression is most beneficial at the higher strain in Figure 13. Estimation noise here comes mostly from $\sigma_{\hat{F}}^{2}$, so EPZS_L1 performs much better when a high level of log compression is applied. CCM L1 is also improved by high log compression, although it peaks at a relatively low value of $c$. The AMC algorithms are also improved by slight log compression, indicating that the AMC formulae are less accurate at high strain, so a combination of AMC and log compression yields the lowest location variance. However, the AMC algorithms have considerably higher peaks than the uncorrected algorithms, so performance convergence as $c \rightarrow \infty$ represents a significant reduction in $\mathrm{SNR}_{e}$. The choice of $c=10^{3}$ for subsequent tests reflects a balance between the EPZS_L1 optima at $0.5 \%$ and $4 \%$ strain.

The $\mathrm{SNR}_{e}$-strain characteristics in Figures 14-16 further demonstrate the advantage of applying AMC. It yields the best performance in both EPZS and CCM families of estimators. The uncorrected EPZS and CCM curves again reach a plateau in the region where $\sigma_{\hat{F}}^{2}$ dominates, as predicted by Equation 7. It is also interesting to note that the AMC curves peak at lower strains than the other algorithms, which follows from the combined effects of AMC becoming less accurate at high strains and $\sigma_{\hat{D}}^{2}$ becoming more important as the level of signal decorrelation increases. In the case of EPZS_A, AMC is precisely accurate for small strains, but it diverges from the correct estimation location at higher strains where errors in the assumptions of the derivation become increasingly significant. The hybrid algorithm, EPZS_LA is the best at $4 \%$ strain, so the combination of AMC with moderate log compression may be the best noise suppression strategy at high strains.

Figure 16 compares the best estimators from each family of algorithms. EPZS_A has the best performance at most strains by a large margin. At low strains the worst algorithm is ASE. This may indicate that the signal stretching technique is inherently more noisy, although at higher strains its advantages are the absence of the AM effect and lower signal decorrelation. Therefore, ASE outperforms CCM_A for strain $>2 \%$, at $4 \%$ it also outperforms EPZS_A, and the gradient of the curve is still positive, so ASE may offer further performance benefits at yet higher strains. However, it is likely that the main advantage of ASE is the relative independence of performance and window length. On the other hand, we have already seen in Figure 9 that EPZS_A outperforms ASE by a large margin if the optimal window length is selected.

Images from real ultrasound scans in Figures 17-20 provide further evidence of the comparative properties of these algorithms. In general, the EPZS_A images are the least noisy, while EPZS_L1 and EPZS_L2 are more or less noisy than EPZS depending on the local strain (c.f. Figures 11-12). These images also demonstrate the importance of AM correction when AM artefacts correlate with features in the B-scans. It is evident in Figure 17 that the AM effect has distorted the shapes of features in the EPZS image, particularly in the attenuation shadow below the olive. Figure 18 shows a more extreme example. The specular reflection is of unknown origin - possibly a crack has developed in the gelatin matrix. It causes severe distortion of EPZS, where the dark patch in Figure 18b looks like a low strain planar inclusion. However, EPZS_L2 is provably unaffected by the AM effect, so real tissue features must also appear in Figure 18d. The dark patch is absent, proving that it is actually an artefact. A mild artefact is also observed with EPZS_A in Figure 18e, where the local sparseness of estimation locations around the reflection causes a textural change in its vicinity.

The in vivo images in Figures 19 and 20 demonstrate that AM artefacts often occur in scans of real human tissue - isoechoicity is rarely a feature of salient scan planes. The male breast in Figure 19 has an appreciably different strain image with EPZS compared to the other algorithms. A bright band at the top of the lower section reappears as a zero-strain band in Figure 19b, but this is an artefact, absent from Figures 19c-e. Many similar artefacts are present in the calf scan of Figure 20. This is extremely anisoechoic, and comparison between Figure 20b and Figures 20c-e shows that all of the main features in the EPZS image are artefacts. 


\subsection{Conclusion}

The AM effect has been theoretically introduced and empirically investigated. A new technique called AMC has been derived for the enhancement of ultrasonic displacement and strain estimates. Simulation, in vitro and in vivo results show a substantial reduction in the level of estimation noise. However, it is always possible to reduce noise by applying filters, thereby sacrificing spatial resolution in order to boost $\mathrm{SNR}_{e}$. It is likely in practice, therefore, that the main impact of AMC will be an improvement in spatial resolution, and AMC can be extended trivially to enhance strain imaging in 2D or 3D if required. The ultimate limiting factor in ultrasonic displacement and strain estimation will be the limited bandwidth of RF ultrasound signals, i.e., the point spread function is not an impulse. This means that even if signal displacements were tracked perfectly, there would be a residual error between those displacements and the actual tissue motion. Developments in ultrasound deconvolution for enhanced ultrasonic resolution may eventually play an important role in high quality ultrasonic strain imaging [23].

It should not be overlooked that the AM effect is likely to feature in many displacement estimation problems where other types of signals are used, such as standard video data. In principle, AMC is a modality independent technique for enhanced tracking of small motions. There are likely to be applications in a wide range of research fields - cosmology, for example - although it is also possible that in some of these areas equivalent techniques may already have been developed independently.

Regarding immediate developments in ultrasonic strain imaging, when AMC is applied with regularly spaced windows of a fixed length this leads to variable spacing of the estimation locations. It will be necessary to investigate intelligent algorithms for automatically varying the length and spacing of the windows to maintain spatial resolution with AMC, or to achieve a balance between spatial resolution and estimation noise according to an appropriate cost function.

Another limitation of AMC as presented is the assumption of locally constant strain. Estimation noise will increase when the second derivative of displacement is non-zero within any particular window. The same limitation applies to ASE. These first order corrections are already very useful, but it should be possible to derive superior AMC formulae by exploiting correlations between the errors in overlapping windows. This is a topic for further investigation. It is also noted that AMC was less accurate when applied to CCM, even for uniform strains, so the derivation of a superior AMC for CCM presents another possible avenue for future work.

On the other hand, the secondary finding of this study is the relative ease with which correction techniques can be developed for phase-based methods. Log compression in both its moderate and limiting forms has been demonstrated to be far more useful with phase (EPZS L1 and EPZS_L2) than with the correlation coefficient (CCM_L1 and CCM_L2). The retention of phase information, regardless of how far the amplitude is compressed, makes phase-based methods more robust. Indeed, in some tests EPZS_L2 has been one of the most successful algorithms. This appears to conflict with the amplitude modulation benefits predicted in Section 2.5, but EPZSL2 actually does exhibit a higher level of displacement estimation error $\left(\sigma_{\hat{D}}^{2}\right)$. Crucially EPZS_L2 is unaffected by location errors $\left(\sigma_{\hat{F}}^{2}\right)$. At high strains $\sigma_{\hat{F}}^{2}$ is often the primary source of error, so EPZSL2 outperforms some of the other estimators. This is especially interesting, since there are computational advantages if all of the amplitude information can be discarded.

In conclusion, we have revisited log compression and found that EPZS_L2 is a good algorithm for imaging high strains, potentially at extremely low computational cost. However, it is inferior to the EPZS_A algorithm incorporating AMC. EPZS_A is marginally more computationally expensive, but it is still suitable for real-time strain imaging. It outperforms all of the other algorithms tested in this study throughout the typical range of strains encountered in practical ultrasonic strain imaging systems.

\section{Acknowledgements}

James $\mathrm{Ng}$ measured the point spread function of the Dynamic Imaging Diasus $5-10 \mathrm{MHz}$ probe. 


\section{Appendices}

\section{A Benefits of amplitude modulation}

We analyse a generic displacement estimator, motivated by the actual properties of phase-based methods. We assume that a window of arbitrary length produces an unbiased displacement estimate. The shortest possible window covers one RF sample, producing a displacement estimate, $\breve{d}$. The estimation variance, $\sigma_{\breve{d}}^{2}$, is inversely proportional to the local ultrasonic SNR; this assumption follows the Cramer-Rao lower bound for displacement estimation variance [3].

$$
\sigma_{\breve{d}}^{2}(t)=\frac{C_{1}}{\operatorname{SNR}(t)}
$$

We assume a simple model for RF signals during a strain imaging ultrasound scan. An underlying signal, $r$, is present in both the pre- and post-deformation signals, $r_{1}$ and $r_{2}$, but these are recorded in the presence of additive noise.

$$
\begin{aligned}
r_{1}(t) & =r(t)+n_{1}(t) \\
r_{2}(t+d(t)) & =r(t)+n_{2}(t+d(t))
\end{aligned}
$$

$n_{1}$ and $n_{2}$ have zero mean, with power $\sigma_{n}^{2}$. They are mutually uncorrelated, and both noise signals are uncorrelated with $r$. In general $n_{1}$ and $n_{2}$ consist not only of electronic noise - other sources of uncorrelated signal components include morphological changes to the speckle pattern [21] and non-axial scatterer motion. The SNR can be expressed in terms of these signal components.

$$
\operatorname{SNR}(t)=\frac{r(t)^{2}}{\frac{1}{2}\left(n_{1}(t)^{2}+n_{2}(t+d(t))^{2}\right)}
$$

The constant of proportionality in Equation 43, $C_{1}$, must be a large number, since the short windows produce inaccurate estimates. However, the generic estimator actually uses longer windows, yielding a weighted average of the single-sample estimates.

$$
\hat{d}_{n}=\frac{\sum_{t=n \Delta t}^{n \Delta t+T} W(t) \check{d}(t)}{\sum_{t=n \Delta t}^{n \Delta t+T} W(t)}
$$

$\hat{d}_{n}$ is the final displacement estimate at window $n$, and $W(t)$ is the weighting for estimate $\check{d}(t)$. If errors in the single-sample estimates are mutually uncorrelated, then the variance of the overall estimate is as follows.

$$
\sigma_{\hat{d}_{n}}^{2}=\frac{\sum_{t=n \Delta t}^{n \Delta t+T} W(t)^{2} \sigma_{\breve{d}}^{2}(t)}{\left(\sum_{t=n \Delta t}^{n \Delta t+T} W(t)\right)^{2}}
$$

This can be minimised by choosing $W$ as follows, where $C_{2}$ is an arbitrary constant.

$$
W(t)=\frac{C_{2}}{\sigma_{\breve{d}}^{2}(t)}=\frac{C_{2} \operatorname{SNR}(t)}{C_{1}}
$$

The implications of this result are not immediately obvious, since $\operatorname{SNR}(t)$ is an unknown quantity. However, the expected error is minimised by choosing $W$ according to the expected value of the local SNR, given the information that is available. We require the statistical expectation of the RHS in Equation 46.

$$
\begin{aligned}
\mathrm{E}[\mathrm{SNR}(t)] & =\mathrm{E}\left[\frac{r(t)^{2}}{\frac{1}{2}\left(n_{1}(t)^{2}+n_{2}(t+d(t))^{2}\right)}\right] \\
& =\mathrm{E}\left[r(t)^{2}\right] \times \mathrm{E}\left[2\left(n_{1}(t)^{2}+n_{2}(t+d(t))^{2}\right)^{-1}\right]
\end{aligned}
$$


The expected noise term is assumed constant $\left(C_{3}\right)$. More sophisticated noise estimates are possible if assumptions can be made about the statistical properties of the noise source, but we restrict ourselves to the most general approach (note, $\mathrm{E}\left[x^{-1}\right] \neq \mathrm{E}[x]^{-1}$, so $C_{3} \neq \sigma_{n}^{-2}$ ).

$$
\mathrm{E}[\operatorname{SNR}(t)]=C_{3} \mathrm{E}\left[r(t)^{2}\right]
$$

Since the noise is uncorrelated and the displacement estimate is assumed to be unbiased, the expectation of the local cross power of the recorded signals is equal to the expected signal power.

$$
\begin{aligned}
\mathrm{E}\left[r_{1}(t) r_{2}(t\right. & \left.\left.+\hat{d}_{n}\right)\right]=\mathrm{E}\left[r_{1}(t) r_{2}(t+d(t))\right] \\
& =\mathrm{E}\left[\left(r(t)+n_{1}(t)\right)\left(r(t)+n_{2}(t+d(t))\right)\right] \\
& =\mathrm{E}\left[r(t)^{2}\right]+\mathrm{E}\left[r(t) n_{1}(t)\right]+\mathrm{E}\left[r(t) n_{2}(t+d(t))\right]+\mathrm{E}\left[n_{1}(t) n_{2}(t+d(t))\right] \\
& =\mathrm{E}\left[r(t)^{2}\right]
\end{aligned}
$$

The cross power can therefore be taken as an estimate of the signal power. By combining the results of Equations 49, 52 and 56, it emerges that the optimal weighting for each single-sample displacement estimate can be evaluated. In the following expression $C_{4}$ is an arbitrary constant.

$$
W(t)=C_{4} r_{1}(t) r_{2}\left(t+\hat{d}_{n}\right)
$$

Weighting by this formula minimises the expected value of $\sigma_{\hat{d}}^{2}$.

\section{References}

[1] S. K. Alam, J. Ophir, and E. E. Konofagou. An adaptive strain estimator for elastography. IEEE Transactions on Ultrasonics, Ferroelectrics, and Frequency Control, 45(2):461-472, March 1998.

[2] J. Bercoff, M. Tanter, and M. Fink. Supersonic shear imaging: a new technique for soft tissue elasticity mapping. IEEE Transactions on Ultrasonics, Ferroelectrics, and Frequency Control, 51(4):396-409, April 2004.

[3] G. C. Carter. Coherence and time delay estimation. Proceedings of the IEEE, 75(2):236-255, 1987.

[4] I. Céspedes and J. Ophir. Reduction of image noise in elastography. Ultrasonic Imaging, 15:89-102, 1993.

[5] X. Chen, M. J. Zohdy, S. Y. Emelianov, and M. O'Donnell. Lateral speckle tracking using synthetic lateral phase. IEEE Transactions on Ultrasonics, Ferroelectrics, and Frequency Control, 51(5):540-550, May 2004.

[6] C. L. de Korte, A. F. W. van der Steen, E. I. Céspedes, and G. Pasterkamp. Intravascular ultrasound elastography in human arteries: experience in vitro. Ultrasound in Medicine and Biology, 24(3):401-408, 1998.

[7] B. S. Garra, E. I. Céspedes, J. Ophir, S. R. Spratt, R. A. Zuurbier, C. M. Magnant, and M. F. Pennanen. Elastography of breast lesions: initial clinical results. Radiology, 202(1):79-86, January 1997.

[8] J. Gennisson, T. Baldeweck, M. Tanter, S. Catheline, M. Fink, L. Sandrin, C. Cornillon, and B. Querleux. Assessment of elastic parameters of human skin using dynamic elastography. IEEE Transactions on Ultrasonics, Ferroelectrics, and Frequency Control, 51(8):980-989, August 2004.

[9] T. J. Hall, Y. Zhu, and C. S. Spalding. In vivo real-time freehand palpation imaging. Ultrasound in Medicine and Biology, 29(3):427-435, 2003. 
[10] L. Han, J. A. Noble, and M. Burcher. A novel ultrasound indentation system for measuring biomechanical properties of in vivo soft tissue. Ultrasound in Medicine and Biology, 29(6):813$823,2003$.

[11] J. A. Jensen. Field: a program for simulating ultrasound systems. In Proceedings of the 10th Nordic-Baltic Conference on Biomedical Imaging, volume 4, pages 351-353, 1996.

[12] F. Kallel and M. Bertrand. Tissue elasticity reconstruction using linear perturbation method. IEEE Transactions on Medical Imaging, 15(3):299-313, June 1996.

[13] F. Kallel and J. Ophir. A least-squares strain estimator for elastography. Ultrasonic Imaging, 19:195-208, 1997.

[14] F. Kallel, T. Vargese, J. Ophir, and M. Bilgen. The nonstationary strain filter in elastography: Part ii. lateral and elevational decorrelation. Ultrasound in Medicine and Biology, 23(9):1357$1369,1997$.

[15] K. Kaluzynski, X. Chen, S. Y. Emelianov, S. R. Skovoroda, and M. O'Donnell. Strain rate imaging using two-dimensional speckle tracking. IEEE Transactions on Ultrasonics, Ferroelectrics, and Frequency Control, 48(4):1111-1123, July 2001.

[16] E. E Konofagou, T. Varghese, and J. Ophir. Theoretical bounds on the estimation of transverse displacement, transverse strain and Poisson's ratio in elastography. Ultrasonic Imaging, 22(3):153-177, 2000.

[17] S. Langeland, J. d'Hooge, H. Torp, B. Bijnens, and P. Suetens. Comparison of time-domain displacement estimators for two-dimensional RF tracking. Ultrasound in Medicine and Biology, 29(8):1177-1186, 2003.

[18] R. M. Lerner, S. R. Huang, and K. J. Parker. Sonoelasticity images derived from ultrasound signals in mechanically vibrated tissues. Ultrasound in Medicine and Biology, 16:231-239, 1990.

[19] J. E. Lindop, G. M. Treece, A. H. Gee, and R. W. Prager. 3D elastography using freehand ultrasound. 2006. Ultrasound in Medicine and Biology, in press.

[20] M. A. Lubinski, S. Y. Emelianov, and M. O'Donnell. Speckle tracking methods for ultrasonic elasticity imaging using short-time correlation. IEEE Transactions on Ultrasonics, Ferroelectrics, and Frequency Control, 46(1):82-96, January 1999.

[21] R. L. Maurice and M. Bertrand. Lagrangian speckle model and tissue-motion estimation theory. IEEE Transactions on Medical Imaging, 18(7):593-603, July 1999.

[22] M. I. Miga. A new approach to elastography using mutual information and finite elements. Physics in Medicine and Biology, 48(1):467-480, January 2003.

[23] J. K. H. Ng, R. W. Prager, N. G. Kingsbury, G. M. Treece, and A. H. Gee. An iterative, wavelet-based deconvolution algorithm for the restoration of ultrasound images in an EM framework. San Diego, California, February 2006. To appear in Proceedings of SPIE Medical Imaging 2006.

[24] R. K. Nightingale, M. L. Palmeri, R. W. Nightingale, and G. E. Trahey. On the feasibility of remote palpation using acoustic radiation force. Journal of the Acoustical Society of America, 110(1):625-634, 2001.

[25] M. O'Donnell, A. R. Skovoroda, B. M. Shapo, and S. Y. Emelianov. Internal displacement and strain imaging using ultrasonic speckle tracking. IEEE Transactions on Ultrasonics, Ferroelectrics, and Frequency Control, 41:314-325, May 1994. 
[26] J. Ophir, I. Céspedes, H. Ponnekanti, Y. Yazdi, and X. Li. Elastography: a quantitative method for imaging the elasticity of biological tissues. Ultrasonic Imaging, 13:111-134, 1991.

[27] K. J. Parker, S. R. Huang, R. A. Musulin, and R. M. Lerner. Tissue response to mechanical vibrations for sonoelasticity imaging. Ultrasound in Medicine and Biology, 16:241-246, 1990.

[28] A. Pesavento and A. Lorenz. Real time strain imaging - a new ultrasonic method for cancer detection: first study results. In Proceedings of IEEE Ultrasonics Symposium 2001, pages 1647-1652, October 2001.

[29] A. Pesavento, C. Perrey, M. Krueger, and H. Ermert. A time efficient and accurate strain estimation concept for ultrasonic elastography using iterative phase zero estimation. IEEE Transactions on Ultrasonics, Ferroelectrics, and Frequency Control, 46(5):1057-1067, September 1999 .

[30] J. G. Proakis and D. G. Manolakis. Digital signal processing: principles, algorithms and applications. Upper Saddle River, third edition, 1996.

[31] R. Righetti, J. Ophir, and P. Ktonas. Axial resolution in elastography. Ultrasound in Medicine and Biology, 28(1):101-113, 2002.

[32] L. Sandrin, M. Tanter, S. Catheline, and M. Fink. Shear modulus imaging with 2-D transient elastography. IEEE Transactions on Ultrasonics, Ferroelectrics, and Frequency Control, 49(4):426-435, April 2002.

[33] S. Srinivasan, F. Kallel, R. Souchon, and J. Ophir. Analysis of an adaptive strain estimation technique in elastography. Ultrasonic Imaging, 24:109-118, 2002.

[34] S. Srinivasan and J. Ophir. A zero-crossing strain estimator for elastography. Ultrasound in Medicine and Biology, 29(2):227-238, 2003.

[35] S. Srinivasan, J. Ophir, and S. K. Alam. Theoretical derivation of SNR, CNR and spatial resolution for a local adaptive strain estimator for elastography. Ultrasound in Medicine and Biology, 30(9):1185-1197, 2004.

[36] T. Varghese and J. Ophir. The nonstationary strain filter in elastography: Part i. frequency dependent attenuation. Ultrasound in Medicine and Biology, 23(9):1343-1356, 1997.

[37] T. Varghese and J. Ophir. A theoretical framework for performance characterization of elastography: the strain filter. IEEE Transactions on Ultrasonics, Ferroelectrics, and Frequency Control, 44(1):164-172, January 1997.

[38] T. Varghese and J. Ophir. Characterisation of elastographic noise using the envelope of echo signals. Ultrasound in Medicine and Biology, 24(4):543-555, 1998.

[39] F. Viola and W. F. Walker. A comparison of the performance of time-delay estimators in medical ultrasound. IEEE Transactions on Ultrasonics, Ferroelectrics, and Frequency Control, 50(4):392-401, April 2003.

[40] M. Vogt and H. Ermert. Development and evaluation of a high-frequency ultrasound-based system for in vivo strain imaging of the skin. IEEE Transactions on Ultrasonics, Ferroelectrics, and Frequency Control, 52(3):375-385, March 2005.

[41] W. F. Walker and G. E. Trahey. A fundamental limit on the performance of correlation based phase correction and flow estimation techniques. IEEE Transactions on Ultrasonics, Ferroelectrics, and Frequency Control, 41(5):644-654, September 1994.

[42] W. F. Walker and G. E. Trahey. A fundamental limit on delay estimation using partially correlated speckle signals. IEEE Transactions on Ultrasonics, Ferroelectrics, and Frequency Control, 42(2):301-308, March 1995. 
[43] Y. Zhu and T. J. Hall. A modified block matching method for real-time freehand strain imaging. Ultrasonic Imaging, 24:161-176, 2002. 